\title{
Article
}

\section{Radial kinematics of brightest cluster galaxies}

Loubser, S. I., Sansom, A. E., Sanchez-blazquez, P., Soechting, I. K. and Bromage, G. E.

Available at http://clok.uclan.ac.uk/4743/

Loubser, S. I., Sansom, A. E. ORCID: 0000-0002-2782-7388, Sanchez-blazquez, P., Soechting, I. K. and Bromage, G. E. (2008) Radial kinematics of brightest cluster galaxies. Monthly Notices of the Royal Astronomical Society, 391 (3). pp. 1009-1028. ISSN 00358711

It is advisable to refer to the publisher's version if you intend to cite from the work. http://dx.doi.org/10.1111/j.1365-2966.2008.13813.x

For more information about UCLan's research in this area go to http://www.uclan.ac.uk/researchgroups/ and search for <name of research Group>.

For information about Research generally at UCLan please go to http://www.uclan.ac.uk/research/

All outputs in CLoK are protected by Intellectual Property Rights law, including Copyright law. Copyright, IPR and Moral Rights for the works on this site are retained by the individual authors and/or other copyright owners. Terms and conditions for use of this material are defined in the policies page.

\section{CLoK}

Central Lancashire online Knowledge www.clok.uclan.ac.uk

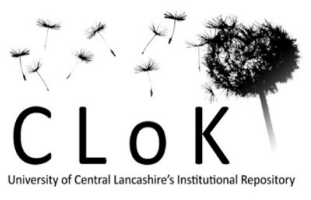




\title{
Radial kinematics of brightest cluster galaxies
}

\author{
S. I. Loubser, ${ }^{1 \star}$ A. E. Sansom, ${ }^{1}$ P. Sánchez-Blázquez, ${ }^{1}$ I. K. Soechting ${ }^{2}$ \\ and G. E. Bromage ${ }^{1}$ \\ ${ }^{1}$ Centre for Astrophysics, University of Central Lancashire, Preston PR1 $2 \mathrm{HE}$ \\ ${ }^{2}$ Oxford Astrophysics, Department of Physics, University of Oxford, Oxford OX1 3RH
}

Accepted 2008 August 10. Received 2008 August 05; in original form 2008 February 01

\begin{abstract}
This is the first of a series of papers devoted to the investigation of a large sample of brightest cluster galaxies (BCGs), their kinematic and stellar population properties, and the relationships between those and the properties of the cluster. We have obtained high signal-to-noise ratio, long-slit spectra of these galaxies with Gemini and William Herschel Telescope with the primary purpose of investigating their stellar population properties. This paper describes the selection methods and criteria used to compile a new sample of galaxies, concentrating on BCGs previously classified as containing a halo ( $\mathrm{cD}$ galaxies), together with the observations and data reduction. Here, we present the full sample of galaxies, and the measurement and interpretation of the radial velocity and velocity dispersion profiles of 41 BCGs. We find clear rotation curves for a number of these giant galaxies. In particular, we find rapid rotation $\left(>100 \mathrm{~km} \mathrm{~s}^{-1}\right)$ for two BCGs, NGC 6034 and 7768, indicating that it is unlikely that they formed through dissipationless mergers. Velocity substructure in the form of kinematically decoupled cores is detected in 12 galaxies, and we find five galaxies with velocity dispersion increasing with radius. The amount of rotation, the velocity substructure and the position of BCGs on the anisotropy-luminosity diagram are very similar to those of 'ordinary' giant ellipticals in high-density environments.
\end{abstract}

Key words: galaxies: elliptical and lenticular, $\mathrm{cD}$ - galaxies: kinematics and dynamics.

\section{INTRODUCTION}

The galaxies in the centres of clusters are unique. They are usually the dominant, brightest and most massive galaxies in their clusters. A wealth of imaging data has been accumulated for these intriguing objects (Malumuth \& Kirshner 1985; Schombert 1986, 1987, 1988; Postman \& Lauer 1995; Collins \& Mann 1998; Brough et al. 2002; Laine et al. 2003); however the spectroscopic data are limited to very small samples or narrow wavelength coverage.

Tonry (1984), Tonry (1985), Gorgas, Efstathiou \& AragonSalamanca (1990), Fisher, Illingworth \& Franx (1995a), Fisher, Franx \& Illingworth (1995b), Cardiel, Gorgas \& Aragon-Salamanca (1998) and Carter, Bridges \& Hau (1999) each investigated 18 or less galaxies, and, moreover, measured only three or less indices. Brough et al. (2007) used a wide wavelength range but included only three brightest cluster galaxies (BCGs). Von der Linden et al. (2007) studied BCGs from the Sloan Digital Sky Survey, however the spatial information is lacking since fibres were used.

Among BCGs, there exists a special class of galaxies catalogued as $\mathrm{cD}$ galaxies, although there exists a great deal of confusion over the exact meaning of the classifications $\mathrm{gE}, \mathrm{D}$ and $\mathrm{cD}$. Matthews, Morgan \& Schmidt (1964) outlined the following definition: 'D galaxies have an elliptical-like nucleus surrounded by an extensive envelope. The supergiant $\mathrm{D}$ galaxies observed near the centre of a number of Abell's rich clusters have diameters three to four times as great as the ordinary lenticulars in the same clusters. These very large D galaxies observed in clusters are given the prefix ' $c$ ', in a manner similar to the notation for supergiant stars in stellar spectroscopy'. Later, Schombert (1987) defined gE galaxies as distinct from other early-type galaxies by their large size; D galaxies as being $\mathrm{gE}$ galaxies with a shallow surface brightness profile slope and cD galaxies as D galaxies with large extended stellar haloes. The last are also more diffuse than normal ellipticals (Schombert 1986). Because of the confusion surrounding the definition of $\mathrm{D}$ galaxies, and the fact that they are rarely regarded as a separate type of object in the modern literature, this study will only refer to $\mathrm{cD}$ and non-cD BCGs (i.e. BCGs containing a halo or not). For BCGs, we adopt the definition to comply with recent literature (e.g. Von der Linden et al. 2007), where BCG refers to the central, dominant galaxy in a cluster. For a small fraction of clusters, the BCG might not strictly be the brightest galaxy in the cluster.

Approximately 20 per cent of the rich clusters of galaxies contain a dominant central cD galaxy (Dressler 1984; Oegerle \& Hill 2001),

^E-mail: siloubser@uclan.ac.uk (SIL) 
although they can be found in poor clusters as well (Giacintucci et al. 2007). Some clusters have more than one cD galaxy, but a cD galaxy is always the dominant member of a local subcluster. The surface-brightness profiles of $\mathrm{cD}$ galaxies are displaced above the de Vaucouleurs law (de Vaucouleurs 1948) at large radii. The break in the cD galaxy surface-brightness profile typically occurs between 24 and 26 mag $\operatorname{arcsec}^{-2}$ in the $V$ band (Sarazin 1988). The interpretation of this deviation is that the galaxy is embedded in an extensive luminous stellar halo. Three main theories have been proposed over the last four decades to explain the properties of BCGs, and in particular $\mathrm{cD}$ galaxies.

Theory 1. In the first theory, BCG formation is caused by the presence of cooling flows in clusters of galaxies (Cowie \& Binney 1977). Cooling flow clusters are common in the local Universe, and BCGs are most often found at the centres of these systems (Edwards et al. 2007). If the central cluster density is high enough, intracluster gas can condense and form stars at the bottom of the potential well. Observations that support this idea are blue- and ultraviolet-colour excesses observed in the central galaxy of Abell 1795 (indicative of star formation) by McNamara et al. (1996) and molecular gas detected in 10 out of 32 central cluster galaxies by Salomé \& Combes (2003). Cardiel et al. (1998) obtained radial gradients for the $\mathrm{D}_{4000}$ and $\mathrm{Mg}_{2}$ spectral features in 11 central cluster galaxies. Their observations were consistent with an evolutionary sequence in which radio-triggered star formation bursts take place several times during the lifetime of the cooling flow in the centre of the cluster. However, McNamara \& O'Connell (1992) find only small colour anomalies with small amplitudes, implying star formation rates that account for at most a few per cent of the material that is cooling and accreting on to the central galaxy.

More recently, XMM-Newton observations showed that the $\mathrm{X}$-ray gas in cluster centres does not cool significantly below a threshold temperature of $k T \sim 1-2 \mathrm{keV}$ (Jordán 2004, and references therein). The central cluster galaxies often host radio-loud active galactic nuclei (AGN), which may account for the necessary heating to counteract radiative cooling (Von der Linden et al. 2007). Although BCGs are probably not completely formed in the cooling flows, the flows play an important role in regulating the rate at which gas cools at the centres of groups and clusters.

Theory 2. The second theory was proposed by Merritt (1983) and suggests that the essential properties of BCGs, and in particular those with haloes, are determined when the clusters collapse (primordial origin). Thereafter, frequent mergers of galaxies would be inhibited by the relatively high velocities between galaxies. Merritt (1983) argued that all galaxies had large haloes early in the life of the cluster. These haloes were then removed by the mean cluster tidal field during the initial collapse and returned to the cluster potential, except for the central member which remained unaffected because of its special position with respect to the cluster potential.

Theory 3. The third, and most widely accepted, theory is in the context of the $\Lambda$ cold dark matter $(\Lambda \mathrm{CDM})$ cosmology and relates the formation of the central galaxy to mergings with or captures of less massive galaxies, and is known as 'galactic cannibalism'. It was first proposed by Ostriker \& Tremaine (1975) and developed by Ostriker \& Hausman (1977).

The most complete quantitative prediction of the formation of BCGs in the now standard CDM model of structure formation is by De Lucia \& Blaizot (2007). They used $N$-body and semi-analytic techniques to study the formation and evolution of BCGs and found that, in a model where cooling flows are suppressed at late times by AGN activity, the stars of BCGs are formed very early (50 per cent at $z \sim 5$ and 80 per cent at $z \sim 3$ ) and in many small galaxies. They also found that BCGs assemble late: half of their final mass is typically locked up in a single galaxy after $z \sim 0.5$ (illustrated in their fig. 9). A very similar conclusion was reached by Romeo et al. (2008), who performed $N$-body and hydrodynamical simulations of the formation and evolution of galaxy groups and clusters in a $\Lambda \mathrm{CDM}$ cosmology to follow the build up of two clusters and 12 groups. Observationally, Aragon-Salamanca, Baugh \& Kauffmann (1998) examined the $K$-band Hubble diagram for BCGs up to a redshift of $z=1$. They found that the BCGs had grown by a factor of 2 to 4 since $z=1$. Brough et al. (2002) found a similar result but discovered that the mass growth depended on the X-ray luminosity of the host cluster. They found that BCGs in high X-ray luminosity clusters showed no mass accretion since $z=1$ as opposed to BCGs in low X-ray luminosity clusters which grew by a factor of 4 . However, the recent near-infrared photometric study of 42 BCGs by Whiley et al. (2008) in the $0.2<z<1$ range contradicts this. They studied the colour and rest-frame $K$-band luminosity evolution of BCGs and found it to be in good agreement with the population synthesis models of stellar populations which formed at $z \sim 2$ and evolved passively thereafter.

Using the Millennium Simulation, De Lucia et al. (2006) studied how formation histories, ages and metallicities of elliptical galaxies depend on environment and on stellar mass. Their fig. 9 shows the effective number of progenitors of early-type galaxies as a function of galaxy stellar mass. The number of effective progenitors is less than two for galaxies up to stellar masses of $\simeq 10^{11} \mathrm{M}_{\odot}$. This function suddenly increases up to the value of approximately five effective progenitors for the mass of a typical BCG.

A related theory, called tidal stripping, was first proposed by Gallagher \& Ostriker (1972). Cluster galaxies that pass near the gravitational centre of the cluster may be stripped of some of their material by the tidal forces from the cluster potential or the central galaxy potential. The stripped material falls to the centre of the potential well, and could contribute to the observed haloes of $\mathrm{cD}$ galaxies. The most massive galaxies surrounding the central galaxy would be preferentially depleted as they are most strongly affected by dynamical friction (Jordán 2004). The difference between stripping and primordial origin is that stripping (and $\mathrm{cD}$ halo formation) begins after the cluster collapse, whereas primordial origin assumes that the tidal events occur before the collapse, and that the $\mathrm{cD}$ halo is not a consequence of tidal stripping (Schombert 1988).

The observation of multiple nuclei in central galaxies favours the cannibalism theory (Postman \& Lauer 1995). Tonry (1984) observed the velocity and velocity dispersion profiles of NGC 6166 and 7720 and their multiple nuclei. He found that the stellar velocity dispersion of the central galaxy demonstrated that the multiple nuclei are not following circular orbits. This was followed by a bigger sample of 14 multiple nuclei BCGs for which the redshifts and stellar velocity dispersions are presented in Tonry (1985). Yamada et al. (2002) showed that the BCG in a cluster at $z=1.26$ is composed of two distinct subunits that are likely to fully merge on a time-scale of $10^{8}$ years. Jordán (2004) studied the globular cluster systems in BCGs with confirmed haloes from Hubble Space Telescope (HST) observations. They concluded that the observed globular cluster metallicity distributions are consistent with those expected if BCGs galaxies form through cannibalism of numerous galaxies and protogalactic fragments that formed their stars and globular clusters before capture and disruption, although they state 
that the cannibalism scenario is not the only possible mechanism to explain these observations. The Jordán (2004) globular cluster data also suggest that BCGs experienced their mergers prior to cluster virialization, yet the presence of tidal streams suggests otherwise (Seigar, Graham \& Jerjen 2007).

Carter \& Metcalfe (1980) and West (1989) showed that the major axis (MA) of BCGs tends to be aligned with the MA of the cluster galaxy distribution. Recent studies of the Coma cluster (Torlina, De Propris \& West 2007) show strong evidence that there are no other large-scale galaxy alignments other than for the BCGs. This also confirms that BCGs form via mechanism related to collimated infall of galaxies along the filaments and the growth of the cluster from the surrounding large-scale structure (Boylan-Kolchin, Ma \& Quataert 2006).

In the CDM cosmology, it is now understood that the local massive cluster galaxies assemble late through the merging of smaller systems. In this picture, cooling flows are the main fuel for galaxy mass growth at high redshift. This source is removed only at low redshifts in group or cluster environments, due to AGN feedback (De Lucia \& Blaizot 2007).

Some of the outstanding issues are: whether BCGs have a different formation mechanism than elliptical galaxies and if the formation of BCGs is controlled by environment. To address these issues the present project was initiated, and long-slit spectra of a large and statistically significant sample of BCGs were obtained. These data provide a set of spectral indices, covering a wide wavelength range. The luminous central galaxies will be contrasted with other early-type galaxies to look for relative differences in their evolution, using features sensitive to stellar population age and abundances of various elements.

The first part of this project entails the determination of the stellar kinematics through derivation of velocity and velocity dispersion profiles. A forthcoming paper will be devoted to the measurement and analysis of the line strengths for this sample of BCGs.

This paper is organized as follows. Section 2 details the sample and the selection criteria, followed by a description of the observations and data reductions in Section 3. The kinematical measurements are described in Section 4, and the individual galaxy kinematic profiles and notes are presented in Section 5. Section 6 discusses the kinematic properties of BCGs as a class, compared to other Hubble types. Conclusions and future work are given in Section 7.

\section{SAMPLE}

The initial intention of this project was to study $\mathrm{cD}$ galaxies in particular. However, the confusion regarding the classification of the $\mathrm{cD}$ galaxies and the lack of deep photometry, which would allow to conclusively distinguish between BCGs with and without a halo, made the sample selection extremely challenging. Aiming at maximizing the completeness of the $\mathrm{cD}$ sample and inclusion of any potential subpopulation, we adopted rather broad selection criteria when restricting an intrinsic BCG sample. We expect to add deep photometric information in future publications, which will allow us to test the presence of a faint halo and discriminate between $\mathrm{cD}$ and non-cD BCGs. For the purpose of this publication, we refer to our sample of 63 galaxies as a BCG sample and present kinematics results for 41 observed members. Two ordinary elliptical galaxies were also observed (one $\mathrm{E}$ and one E/SO) and their kinematics presented here. They will be used as a control sample in this study, where relevant.
The sample selection combines three methods making the best use of available information from literature and astronomical data bases. The three search methods are described below.

Method 1. Two well-known galaxy cluster classification systems that distinguish clusters containing a $\mathrm{cD}$ galaxy in the centre from other galaxy clusters are those of Rood \& Sastry (1971, hereafter RS) and Bautz \& Morgan (1970, hereafter BM). The RS classification is based on the projected distribution of the brightest 10 members, and the BM classification is based on the brightness contrast between first- and second-ranked galaxies (i.e. the slope of the luminosity function at the bright end).

Hoessel, Gunn \& Thuan (1980) derived the BM types for all the nearby Abell clusters of galaxies, which made it possible to obtain a list of all nearby Abell clusters with BM types I and I-II (classified to contain a cD galaxy). Struble \& Rood (1987) compiled a catalogue of the morphological properties of 2712 Abell clusters, derived from a visual inspection of the KPNO photographic plates. This made it possible to obtain a list of Abell clusters with RS cluster classification type cD. The NASA/IPAC Extragalactic Data base (NED) ${ }^{1}$ was used to search for the brightest galaxies close to the centre of each cluster. If the brightest member was classified as either a cD or a D galaxy in NED (in the NED morphology or notes of previous observations), it was included in our list of BCGs. The $\mathrm{RS}$ and/or BM classifications are frequently used as a method to identify possible cD galaxies (e.g. Hill \& Oegerle 1992; Baier \& Wipper 1995; Giacintucci et al. 2007).

Method 2. The second search was carried out by performing an all-sky search in the HyperLEDA ${ }^{2}$ data base for galaxies with the following properties: $\mathrm{T}$ type between -3.7 and -4.3 [the de Vaucouleurs Third Reference Catalogue (RC3) classifies cD galaxies as T type $=-4$ ]; apparent $B$ magnitude brighter than 16; distance closer than $340 \mathrm{Mpc}$ and further than $15^{\circ}$ from the Galactic plane. ${ }^{3}$ It was found that $\mathrm{T}$ type alone is not a reliable classification of galaxy types (in both the RC3 and HyperLeda catalogues), as various low-luminosity galaxies are also categorized as galaxies with $\mathrm{T}$ type $=-4$. This prompted an absolute magnitude cut-off of $M_{\mathrm{B}}=-20$ to be applied throughout the sample selection. The galaxies obtained with this search were added to our list of BCGs if they were classified as $\mathrm{cD}$ galaxies in NED.

Method 3. The third search was undertaken by choosing galaxies with published surface brightness profiles that had a slope consistent with $\mathrm{cD}$ galaxies, and in the case of $\mathrm{cD}$ galaxies broke the de Vaucouleurs $r^{\frac{1}{4}}$ law at large radii. Galaxies were chosen from the series of papers by Schombert $(1986,1987,1988)$ and from Malumuth \& Kirshner (1985). These are all optical photometric studies of the brightest cluster members of clusters. Galaxies with the surface brightness slopes and profiles conforming to the $\mathrm{cD} / \mathrm{D}$ criteria set in Schombert (1987) were included in our list since that forms the basis of most definitions of $\mathrm{cD}$ galaxies.

The complete list of galaxies and their properties is given in Table 1. The following global criteria were applied to the sample: apparent $B$ magnitude brighter than 16 ; distance closer than $340 \mathrm{Mpc}$ and the absolute magnitude cut-off at $M_{B}=-20$. Method 1 delivered 13 galaxies, Method 2 delivered 32 galaxies and Method 3

\footnotetext{
${ }^{1}$ http://nedwww.ipac.caltech.edu/.

${ }^{2} \mathrm{http}: / /$ leda.univ-lyon1.fr/.

${ }^{3} \mathrm{An} H_{0}$ value of $75 \mathrm{~km} \mathrm{~s}^{-1} \mathrm{Mpc}^{-1}$ is assumed throughout this work.
} 
Table 1. The new sample of BCGs, including the two ordinary ellipticals (marked as $\star$ ). Columns $2,3,6-9$ are from the HyperLeda catalogue and 4 and 5 from NED. PA is the position angle of the galaxy MA. Refs 1,2 and 3 correspond to the three search methods for objects (Column 10). Run (Column 11) $1=$ WHT, $2=$ Gemini - N 2006B, $3=$ Gemini - S 2006B, $4=$ Gemini - N 2007A, $5=$ Gemini S2007Aand $6=$ Gemini - S 2007B (the data from Run 6 are not presented in this paper.). Besides the galaxies classified as cD/D in the NED morphology (Column 5), NGC 6034, UGC 05515 and NGC 1129 were classified as cD/D in Schombert (1987); NGC 7012, ESO 146-028, ESO 346-003, ESO 202-043, NGC 3842, PGC 026269 and IC 1634 were classified as $\mathrm{cD} / \mathrm{D}$ in the NED notes by previous observations.

\begin{tabular}{|c|c|c|c|c|c|c|c|c|c|c|}
\hline $\begin{array}{l}\text { Object } \\
\text { name }\end{array}$ & $\alpha(\mathrm{J} 2000)$ & $\delta(\mathrm{J} 2000)$ & Cluster & NED type & T type & $\begin{array}{l}\text { PA } \\
(\mathrm{deg})\end{array}$ & $m_{\mathrm{B}}$ & $M_{\mathrm{B}}$ & Refs & Run \\
\hline IC 1565 & 03926.3 & 064403 & A0076 & $\mathrm{cD} ; \mathrm{E}$ & $-3.9 \pm 1.2$ & - & 14.41 & -21.96 & 2 & \\
\hline UGC 00579 & 05616.2 & -011522 & A0119 & $\mathrm{cD} ; \mathrm{E}$ & $-4.8 \pm 0.4$ & 41 & 14.40 & -22.34 & 3 & 6 \\
\hline ESO 541-013 & 10241.8 & -215255 & A0133 & $\mathrm{cD} ; \mathrm{E}+3$ pec & $-4.0 \pm 0.7$ & 16 & 14.71 & -22.54 & 2 & 6 \\
\hline PGC 004072 & 10850.8 & -152431 & A0151 & $\mathrm{D}$ & $-4.2 \pm 1.0$ & 83 & 14.75 & -22.37 & 2 & 6 \\
\hline IC 1633 & 10955.6 & -455552 & A2877 & $\mathrm{cD} ; \mathrm{E}+1$ & $-3.9 \pm 0.5$ & 97 & 12.57 & -22.60 & 2,3 & 3 \\
\hline IC 1634 & 11102.9 & 173946 & A0154 & MLT SYS & $-4.1 \pm 1.0$ & 153 & 15.27 & -22.64 & 1 & \\
\hline IC 1695 & 12507.6 & 084158 & A0193 & $\mathrm{cD} ; \mathrm{S} ?$ & - & 103 & 14.87 & -21.98 & 1 & \\
\hline NGC 0533 & 12531.5 & 014533 & A0189B & cD;E3: & $-4.8 \pm 0.6$ & 50 & 12.45 & -22.25 & 3 & 6 \\
\hline NGC 0541 & 12544.3 & -012246 & A0194 & cD;SO-: & $-3.9 \pm 0.9$ & 69 & 13.09 & -21.62 & 2 & 6 \\
\hline IC 1733 & 15042.9 & 330455 & A0260 & $\mathrm{cD} ; \mathrm{E}:$ & $-4.9 \pm 0.8$ & - & 14.14 & -22.16 & 1 & \\
\hline NGC 0708 & 15246.3 & 360912 & A0262 & $\mathrm{cD} ; \mathrm{E}$ & $-4.9 \pm 0.4$ & 39 & 13.63 & -20.98 & 3 & \\
\hline UGC 02232 & 24603.9 & 365419 & A0376 & $\mathrm{cD} ; \mathrm{E} / \mathrm{D}$ & $-3.9 \pm 1.3$ & - & 15.50 & -21.64 & 2 & 2 \\
\hline NGC 1129 & 25427.4 & 413446 & AWM7 & $\mathrm{E} ; \mathrm{BrClG}$ & $-4.7 \pm 1.2$ & 73 & 13.34 & -21.67 & 3 & \\
\hline UGC 02450 & 25857.8 & 133459 & A0401 & $\mathrm{cD}$ & $2.8 \pm 5.0$ & 29 & 15.24 & -23.50 & 1 & \\
\hline NGC 1275 & 31948.2 & 413042 & A0426 & cD;pec;NLRG & $-2.2 \pm 1.7$ & 110 & 12.55 & -22.69 & 3 & \\
\hline NGC 1399 & 33829.0 & -352658 & RBS454 & $\mathrm{cD} ; \mathrm{E} 1 \mathrm{pec}$ & $-4.5 \pm 0.5$ & - & 10.42 & -20.81 & 3 & 3 \\
\hline ESO 303-005 & 41358.8 & -380550 & RBS512 & $\mathrm{cD} ?$ & $-4.0 \pm 0.5$ & - & 15.38 & -21.51 & 2 & 3 \\
\hline MCG-02-12-039 & 43337.8 & -131540 & A0496 & $\mathrm{cD} ; \mathrm{E}+?$ & $-3.9 \pm 0.9$ & 180 & 13.95 & -22.50 & 2,3 & 3 \\
\hline ESO 202-043 & 43747.6 & -512523 & A S0479 & $\mathrm{E}+$ & $-3.8 \pm 0.8$ & 133 & 14.45 & -21.72 & 2 & 6 \\
\hline ESO 552-020 & 45452.3 & -180653 & CID 28 & $\mathrm{cD} ; \mathrm{E}+$ & $-3.9 \pm 0.7$ & 148 & 13.54 & -22.51 & 2 & 3 \\
\hline NGC 1713 & 45854.6 & 002920 & CID 27 & $\mathrm{cD} ; \mathrm{E}+$ & $-4.3 \pm 0.6$ & 39 & 13.88 & -20.71 & 2 & 2 \\
\hline UGC 03197 & 45955.8 & 801043 & A0505 & $\mathrm{cD}$ & $-4.9 \pm 0.9$ & 85 & 15.25 & -22.26 & 3 & \\
\hline ESO 488-027 & 54838.5 & -252844 & A0548 & $\mathrm{cD} ; \mathrm{E}+1$ & $-3.8 \pm 0.6$ & 68 & 14.22 & -22.22 & 2 & 3 \\
\hline PGC 025714 & 90832.4 & -093747 & A0754 & $\mathrm{D}$ & $-4.2 \pm 1.4$ & 122 & 14.32 & -23.04 & 2 & 6 \\
\hline PGC 026269 & 91805.7 & -120544 & A0780 & $\left(\mathrm{R}^{\prime}\right) \mathrm{SAO}-: ; \mathrm{BrClGSy} 3$ & $-2.6 \pm 1.0$ & 133 & 14.38 & -22.84 & 1 & 3 \\
\hline NGC 2832 & 91946.9 & 334459 & A0779 & $\mathrm{E}+2: ; \mathrm{cD}$ & $-4.3 \pm 0.6$ & 172 & 12.79 & -22.38 & 2,3 & 2 \\
\hline UGC 05515 & 101338.3 & 005532 & A0957 & E+pec: & $-4.0 \pm 0.7$ & 83 & 14.49 & -22.28 & 2,3 & 5 \\
\hline PGC 030223 & 102026.6 & -063135 & A0978 & $\mathrm{D}$ & $-3.9 \pm 1.2$ & 1 & 15.40 & -21.85 & 2 & 6 \\
\hline NGC 3311 & 103642.9 & -273137 & A1060 & $\mathrm{cD} ; \mathrm{E}+2$ & $-3.3 \pm 1.3$ & - & 12.69 & -21.33 & 1 & 5 \\
\hline NGC 3842 & 114402.2 & 195659 & A1367 & $\mathrm{E} ; \mathrm{BrClG}$ & $-4.8 \pm 0.7$ & 177 & 12.80 & -22.18 & 3 & 1 \\
\hline PGC 044257 & 125711.4 & -172436 & A1644 & $\mathrm{cD} ; \mathrm{E}+4$ & $-4.1 \pm 1.0$ & 44 & 13.50 & -23.54 & 1,2 & 5 \\
\hline NGC 4839 & 125724.2 & 272954 & A1656 & $\mathrm{cD} ; \mathrm{SAO}$ & $-4.0 \pm 0.8$ & 64 & 13.05 & -22.26 & 2,3 & 4 \\
\hline NGC 4874 & 125935.5 & 275736 & A1656 & $\mathrm{cD} ; \mathrm{Di}$ & $-3.7 \pm 0.9$ & 45 & 12.75 & -22.50 & 2,3 & 1 \\
\hline NGC 4884(9) & 130007.9 & 275841 & A1656 & $\mathrm{cD} ; \mathrm{E} 4 ; \mathrm{Db}$ & $-4.3 \pm 0.5$ & 82 & 12.48 & -22.56 & 2 & 1 \\
\hline NGC $4946^{\star}$ & 130529.4 & -433528 & A3526 & $\mathrm{E}+?$ & $-3.8 \pm 0.8$ & 135 & 13.41 & -20.27 & & 5 \\
\hline ESO 444-046 & 132756.9 & -312944 & A3558 & $\mathrm{cD} ; \mathrm{E}+4$ & $-3.7 \pm 1.0$ & 161 & 14.07 & -22.86 & 2,3 & 5 \\
\hline LEDA094683 & 135306.4 & 050859 & A1809 & $\mathrm{cD}$ & $-2.3 \pm 5.0$ & 46 & 15.30 & -22.35 & 1 & 5 \\
\hline GSC555700266 & 140136.4 & -110743 & A1837 & $\mathrm{cD}$ & - & - & 14.59 & - & 1 & 5 \\
\hline NGC 5539 & 141737.8 & 081046 & A1890 & $\mathrm{cD}$ & $0.7 \pm 5.0$ & 39 & 14.92 & -22.62 & 1 & \\
\hline IC 1101 & 151056.1 & 054441 & A2029 & cD;SO-: & $-2.9 \pm 1.1$ & 25 & 15.10 & -23.05 & 3 & 5 \\
\hline UGC 09799 & 151644.6 & 070116 & A2052 & $\mathrm{cD} ; \mathrm{E}$ & $-4.7 \pm 1.3$ & 30 & 14.34 & -21.84 & 3 & \\
\hline PGC 054913 & 152305.3 & 083633 & A2063 & $\mathrm{cD} ; \mathrm{S}$ & $-3.8 \pm 1.6$ & - & 14.71 & -21.46 & 2 & \\
\hline UGC 09958 & 153939.1 & 214658 & A2107 & cD;SO-: & $-2.9 \pm 0.7$ & 98 & 14.76 & -22.00 & 3 & \\
\hline UGC 10012 & 154459.0 & 360636 & A2124 & $\mathrm{cD} ; \mathrm{E}$ & $-4.9 \pm 0.6$ & 143 & 14.54 & -23.15 & 3 & \\
\hline UGC 10143 & 160217.3 & 155828 & A2147 & $\mathrm{cD} ; \mathrm{E}+$ & $-4.0 \pm 0.8$ & 12 & 14.27 & -21.97 & 2,3 & 1 \\
\hline NGC 6034 & 160332.1 & 171155 & A2151 & $\mathrm{E}+$ & $-4.0 \pm 0.4$ & 59 & 14.58 & -21.60 & 2,3 & 1 \\
\hline NGC $6047^{\star}$ & 160509.1 & 174348 & A2151 & $\mathrm{E}+$ & $-3.5 \pm 0.8$ & 117 & 14.58 & -21.45 & & 1 \\
\hline NGC 6086 & 161235.6 & 292906 & A2162 & $\mathrm{cD} ; \mathrm{E}$ & $-4.8 \pm 0.6$ & 3 & 13.81 & -22.19 & 3 & 4 \\
\hline NGC 6160 & 162741.1 & 405537 & A2197 & $\mathrm{cD} ; \mathrm{E}$ & $-4.8 \pm 0.6$ & 72 & 14.17 & -21.70 & 3 & 4 \\
\hline NGC 6166 & 162838.5 & 393304 & A2199 & $\mathrm{cD} ; \mathrm{E}$ & $-4.2 \pm 1.4$ & 38 & 12.88 & -22.92 & 2,3 & 1 \\
\hline NGC 6173 & 162944.9 & 404842 & A2197 & $\mathrm{cD} ; \mathrm{E}$ & $-4.8 \pm 0.6$ & 138 & 13.13 & -22.58 & 3 & 4 \\
\hline NGC 6269 & 165758.1 & 275116 & AWM5 & $\mathrm{cD} ; \mathrm{E}$ & $-4.7 \pm 0.7$ & 80 & 13.38 & -22.98 & 3 & 4 \\
\hline IC 4765 & 184718.5 & -631950 & A S0805 & $\mathrm{cD} ; \mathrm{E}+4$ & $-3.9 \pm 0.5$ & 123 & 12.46 & -21.97 & 2 & 5 \\
\hline NGC 7012 & 210645.8 & -444849 & A S0921 & $\mathrm{E}+4 \mathrm{pec}$ & $-3.9 \pm 0.5$ & 100 & 13.93 & -21.79 & 2 & 3 \\
\hline ESO $146-028$ & 222851.1 & -605255 & RXCJ2228.8-6053 & $E+3$ & $-3.8 \pm 0.8$ & 154 & 13.94 & -22.54 & 2 & 5 \\
\hline ESO $346-003$ & 224922.0 & -372820 & A S 1065 & $E+2$ pec: & $-3.8 \pm 0.8$ & 118 & 14.00 & -21.59 & 2 & 5 \\
\hline NGC 7597 & 231830.3 & 184120 & A2572 & $\mathrm{cD} ; \mathrm{S} ?$ & $-2.0 \pm 0.8$ & 133 & 15.00 & -21.52 & 1 & 2 \\
\hline NGC 7647 & 232357.4 & 164638 & A2589 & $\mathrm{cD} ; \mathrm{E}$ & $-4.7 \pm 0.8$ & 174 & 14.61 & -21.93 & 3 & 1 \\
\hline
\end{tabular}


Table 1 - continued.

\begin{tabular}{|c|c|c|c|c|c|c|c|c|c|c|}
\hline $\begin{array}{l}\text { Object } \\
\text { name }\end{array}$ & $\alpha(\mathrm{J} 2000)$ & $\delta(\mathbf{J} 2000)$ & Cluster & NED type & T type & $\begin{array}{l}\text { PA } \\
(\text { deg })\end{array}$ & $m_{\mathrm{B}}$ & $M_{\mathrm{B}}$ & Refs & Run \\
\hline NGC 7649 & 232420.1 & 143849 & A2593 & $\mathrm{cD} ; \mathrm{E}$ & $-4.6 \pm 1.1$ & 78 & 15.65 & -20.99 & 3 & 2 \\
\hline NGC 7720 & 233829.5 & 270151 & A2634 & $\mathrm{cD} ; \mathrm{E}+\mathrm{pec}:$ & $-4.3 \pm 0.6$ & - & 13.43 & -22.63 & 2 & 1 \\
\hline IC 5358 & 234745.0 & -280826 & A4038 & $c D ; E+4$ pec & $-3.8 \pm 1.1$ & 114 & 13.64 & -22.00 & 2 & 3 \\
\hline NGC 7768 & 235058.3 & 270851 & A2666 & $\mathrm{cD} ; \mathrm{E}$ & $-4.9 \pm 0.5$ & 55 & 13.28 & -22.39 & 3 & 1 \\
\hline
\end{tabular}

contributed 29 galaxies (indicated in Table 1). With 11 of the merged list being duplicates presented from more than one method, this provided an all-sky list of the 63 galaxies.

In summary: these 63 galaxies were classified as $\mathrm{cD} / \mathrm{D}$ in NED (in the morphological classification or in the notes of previous observations) and/or have profiles breaking the $r^{\frac{1}{4}}$ law. With the exception of NGC 4946 and 6047, which are included in the sample as control galaxies but known to be an E and E/SO galaxy, respectively, all galaxies have thus been previously classified in the literature to contain a halo, albeit very inhomogeneously. Despite the confusion regarding the halo classification, all these galaxies are the dominant central galaxies in clusters or groups. If selection methods 1 and 3 above are assumed to be more robust methods of identifying a BCG with a halo, then 67 per cent of the BCG sample are confirmed cD galaxies. 10 galaxies in the BCG sample are in common with the Fisher et al. (1995a) sample of 13 BCGs. For four Abell clusters, the choice of BCG differs from that by Postman \& Lauer (1995). These are: Abell 0194, 0548, 4038 and 2151. In each of the first three cases, the choice of BCG in this sample is more dominant than the BCG in the Postman \& Lauer (1995) sample. In the case of Abell 2151, we followed previous authors (e.g. Aragon-Salamanca et al. 1998) who have chosen NGC 6034 to be the BCG as it has a very prominent $\mathrm{cD}$ halo (Schombert 1987,1988 ) and is close to an $\mathrm{X}$-ray peak.

Some of our clusters are known to have substructure (Rines et al. 2002; Adami et al. 2005) and, in these cases, there may be more than one local X-ray maximum, which can host a dominant galaxy central to that substructure. Hence, according to the definition adopted here, there can be more than one BCG per cluster. In particular, three of the selected BCGs (NGC 4889, 4874 and 4839) are hosted by the Coma cluster, all of them showing pronounced envelopes (Schombert 1987; Andreon et al. 1996). The first two are the dominant galaxies in the cluster (it is not clear which one is the original central galaxy of the main cluster; see e.g. Adami et al. 2005), while NGC 4839 is the dominant galaxy of a group that appears to be infalling into the main cluster (Neumann et al. 2001). Two galaxies (NGC 6173 and 6160) from the cluster Abell 2197 are also included in the sample. These two galaxies are believed to be the dominant galaxies of two main groups in the process of merging (Abell 2197E and Abell 2197W; Schombert 1987).

An internally consistent photometric study of this BCG sample will be done before investigating the properties of the $\mathrm{cD}$ galaxies as a separate class. Liu et al. (2008) measured the extended envelopes of a sample of BCGs using Petrosian profiles (Patel et al. 2006). There is a distinct signature of a plateau in the Petrosian profiles of cD galaxies with an extended stellar halo, which is not present for normal elliptical galaxies. However, they find that the devia- tion of the surface brightness profiles from a single Sérsic profile does not have any sharp transitions. Therefore, it is very difficult to unambiguously separate $\mathrm{cD}$ from non-cD BCGs based on surface brightness profiles alone (Liu et al. 2008), and confirming the presence or absence of $\mathrm{cD}$ haloes depends on choices of profiles fitted to the surface brightness as a function of radius.

In all the conclusions of this study, it will be clearly stated whether it applies to BCGs or includes the two normal elliptical galaxies. The 43 galaxies discussed further in this first paper are indicated in the last column of Table 1 (numbered 1 to 5 according to observing run), and range from $M_{\mathrm{B}}=-23.54$ to -20.71 for the BCGs.

\section{OBSERVATIONS AND DATA REDUCTION}

\subsection{WHT observations}

Spectroscopic observations of 10 of the 43 galaxies presented here (listed in Table 2) were carried out during the period 2006 June 23 to June 26, using the 4.2-m William Herschel Telescope (WHT) equipped with the ISIS double spectrograph mounted at the $f / 11$ Cassegrain focus. The Marconi2 CCD was employed on the red arm and the EEV12 on the blue. The spatial scale of both CCDs was $0.4 \operatorname{arcsec}_{\text {pixel }}^{-1}$ (with $2 \times 2$ binning) and the slit width was 1 arcsec. Comparison spectra were provided by $\mathrm{Cu} / \mathrm{Ar}$ and $\mathrm{Cu} / \mathrm{Ne}$ arcs and the flat-field illumination by a tungsten lamp.

Two different dichroics were used. The $5300 \AA$ dichroic was used for three nights and delivered an unvignetted spectral range of 3900-5460 $\AA$ in the blue arm and 5730-6960 $\AA$ in the red arm. The $6100 \AA$ dichroic was used for one night, delivering an unvignetted spectral range of 4500-6120 $\AA$ in the blue arm and 7950-9600 $\AA$ in the red arm. The exposure times are listed in Table 2. This combination made it possible to observe 29 absorption indices consisting of the original $21 \mathrm{Lick}$ indices from $\mathrm{CN}$ to $\mathrm{TiO}_{2}$ (Burstein et al. 1984; Faber et al. 1985; Worthey et al. 1994), the four $\mathrm{H} \delta$ and $\mathrm{H} \gamma$ indices contributed by Worthey \& Ottaviani (1997), the near-infrared Ca II triplet at $\sim 8600 \AA$ and the $\mathrm{Mg}$ I index. This collection of 29 indices will be referred to as the Lick indices in this study.

Dispersion in the blue arm was $0.90 \AA$ pixel $^{-1}$ (grating R600B) and $0.88 \AA$ pixel $^{-1}$ (grating R600R) in the red. The spectral resolution ranged between 3.3 and $4.0 \AA$, depending on the dichroic and arm used. Seeing was typically better than 1 arcsec, and all necessary calibration frames were obtained. In most cases, the slit was placed on the MA of the galaxy. Exceptions occurred if there was another early-type galaxy (unrelated to the sample) in close proximity, in which case the slit was positioned to go through the centres of both galaxies, to allow a control sample of ellipticals with 
Table 2. Exposure times in each of the two dichroics and PAs for the WHT BCGs. The PA is given as deg $\mathrm{E}$ of N. The half-light radii $\left(r_{\mathrm{e}}\right)$ were calculated from the 2MASS catalogue, except NGC 6166 and NGC 7720 for which they were calculated with the VAUCOUL task in the REDUCEME package. The last column lists the fraction of the effective half-light radii spanned by the radial profiles measured in this work. Table 1 contains more detailed properties for the galaxies.

\begin{tabular}{lcccccccc}
\hline Object & $\begin{array}{c}\text { Exposure } \\
(5300 \AA) \\
(\mathrm{s})\end{array}$ & $\begin{array}{c}\text { Exposure } \\
(6100 \AA) \\
(\mathrm{s})\end{array}$ & $\begin{array}{c}\text { Slit } \\
\text { PA } \\
(\mathrm{deg})\end{array}$ & $\begin{array}{c}\text { Major axis } \\
(\mathrm{deg})\end{array}$ & $\begin{array}{r}r_{\mathrm{e}} \\
(\operatorname{arcsec})\end{array}$ & & $\begin{array}{c}a_{\mathrm{e}} \\
(\operatorname{arcsec})\end{array}$ & $\begin{array}{c}\text { Fraction } \\
\text { times }\end{array}$ \\
\hline NGC 3842 & $6 \times 1200$ & $2 \times 900$ & 5 & 177 & 16.4 & 0.29 & 16.3 & 1.22 \\
NGC 4874 & $6 \times 1200$ & $3 \times 900$ & 82 & 45 & 20.0 & 0.00 & 20.0 & 0.60 \\
NGC 4889 & $6 \times 1200$ & $2 \times 900$ & 80 & 82 & 25.7 & 0.34 & 25.7 & 0.89 \\
NGC 6034 & $6 \times 1200$ & $3 \times 900$ & 50 & 59 & 8.2 & 0.27 & 8.1 & 1.23 \\
NGC 6047 & $3 \times 1200$ & - & 88 & 117 & 7.4 & 0.27 & 7.0 & 0.61 \\
NGC 6166 & $6 \times 1200$ & $3 \times 900$ & 35 & 38 & 22.6 & 0.26 & 22.6 & 0.22 \\
NGC 7647 & $6 \times 1200$ & $3 \times 900$ & 174 & 174 & 11.2 & 0.49 & 11.2 & 1.21 \\
NGC 7720 & $6 \times 1200$ & $3 \times 900$ & 10 & - & 9.3 & 0.19 & 9.3 & 0.65 \\
NGC 7768 & $3 \times 1200$ & $3 \times 900$ & 60 & 55 & 14.7 & 0.19 & 14.7 & 0.82 \\
UGC 10143 & $6 \times 1200$ & $3 \times 900$ & 12 & 12 & 10.5 & 0.41 & 10.5 & 0.65 \\
\hline
\end{tabular}

the same observational setup (two control sample ellipticals were observed but are not used in the present paper.). Five spectrophotometric standard stars were also observed for flux calibration.

A total of 22 Lick calibration stars $(G$ - and $K$-type stars which are considered the main contributors to the visible light in earlytype galaxies) were observed with the $5300 \AA$ dichroic and 10 with the $6100 \AA$ dichroic. A neutral density filter was used for the Lick stars with both dichroics. These stars are used as the templates for velocity dispersion measurements as well as to transform the line-strength indices to the Lick system. Before and/or after every standard star or object spectrum, an arc spectrum and flat-field frame were obtained in the red and blue arm at each telescope pointing, to allow accurate wavelength calibration as well as the removal of the response function of the dichroics.

\subsection{Gemini observations}

The Gemini Multi-object Spectrograph (GMOS) observations were performed in queue scheduling mode. The B600 grating was used (2.7 A resolution) with a 0.5 arcsec slit width to match the observations of the Lick stars described below. Dispersion was $0.914 \AA$ pixel $^{-1}$, and the spatial scale was 0.146 arcsec pixel ${ }^{-1}$ (with $2 \times 2$ binning). 10 galaxies were observed at Gemini South in semester 2006B (2006 August to 2007 January) and five galaxies at Gemini North in the same observing semester. A further 12 galaxies were observed at Gemini South and six at Gemini North in 2007A (2007 February to 2007 August).

Bryan Miller et al. (private communication) performed a series of observing programmes from 2002 to 2004 in which they acquired long-slit spectroscopic data of 'Lick stars'. These are well suited as calibration data for our observing programmes, and our instrumental setup corresponded to the setup used for observations of the Lick stars. Spectral dithering was carried out with two central wavelengths at 5080 and $5120 \AA$ for the Lick stars as well as the galaxy data described here. This is done in order to obtain the full spectrum uninterrupted by the gaps between the three CCDs. The three CCDs mosaicked together delivered a spectral range from 3700 to $6500 \AA$. Exposure times were $2 \times 900 \mathrm{~s}$ at each position, which amounts to an hour (mostly along the MA) for each galaxy, except in the case of ESO 444-046 where only one $900 \mathrm{~s}$ exposure at each position was taken. Imaging sequences $(3 \times 20 \mathrm{~s}$ with the $g$ and $r$ filter $)$ were performed to allow insight into the possible presence and location of dust lanes or gas relative to the slit position. Standard stars were observed for relative flux calibration. The observations were mostly executed in dark time. The seeing typically ranged from 0.6 to 1.2 arcsec, and very rarely went up to 2.2 arcsec. Calibration arc $(\mathrm{CuAr})$ and flat-field spectra were also frequently observed at the two different central wavelengths. General bias frames and imaging flat-field frames were also obtained.

For the Gemini data, information on the slit placements during observations was used to reconstruct the slit position on images of the galaxies. The slit location on the targets was determined using the slit acquisition images and the target positions from the imaging sequences. The images were taken with the same telescope pointing, and used to measure the offsets of the slit position from the galaxy centre (Table 3 ). In the few cases where the same galaxy was observed on two different nights, this was done for both nights. Offsets from the galaxy centres are shown in Table 3. The majority of the galaxies were centred correctly or with very small offsets. There are no offsets indicated for the WHT observations, because these were carried out in visiting observer mode, and all offsets were zero.

\subsection{Data reduction}

All the WHT data reductions were performed with the $\mathrm{IRAF}^{4}$ and STARLINK $^{5}$ reduction packages. The blue and red spectra and spectra taken using the 5300 and $6100 \AA$ dichroics were treated in similar parallel streams of data reduction. Overscan correction, ADU conversion, bias subtraction and trimming of the image edges were done first. Flat-field spectra were normalized by correcting for the lamp response function, and all star and galaxy spectra were appropriately flat-fielded. The dichroics introduced an intermediatefrequency pattern, which varied with the position of the telescope. This was successfully removed by flat-fielding, using the flat-field

\footnotetext{
${ }^{4}$ IRAF is distributed by the National Optical Astronomy Observatories, which are operated by the Association of Universities for Research in Astronomy, Inc., under cooperative agreement with the National Science Foundation.

${ }^{5}$ STARLINK is an interactive reduction and analysis facility that was developed in the UK, funded by the PPARC/STFC Research Council.
} 
Table 3. Galaxies observed with the Gemini-North and South telescopes. Table 1 contains more detailed properties for the galaxies. The PA is given as deg $\mathrm{E}$ of $\mathrm{N}$. The half-light radii $\left(r_{\mathrm{e}}\right)$ were calculated from the 2MASS catalogue, except ESO 303-005 for which it was calculated with the VAUCOUL task in the REDUCEME package. The second last column lists the fraction of the effective half-light radii spanned by the radial profiles measured in this work and the last column lists the offsets as described in the text.

\begin{tabular}{|c|c|c|c|c|c|c|c|}
\hline Object & $\begin{array}{l}\text { Slit } \\
\text { PA }\end{array}$ & Major axis & $\begin{array}{c}r_{\mathrm{e}} \\
(\operatorname{arcsec})\end{array}$ & $\epsilon$ & $\begin{array}{c}a_{\mathrm{e}} \\
(\operatorname{arcsec})\end{array}$ & $\begin{array}{c}\text { Fraction } \\
a_{\mathrm{e}}\end{array}$ & $\begin{array}{c}\text { Offset } \\
(\operatorname{arcsec})\end{array}$ \\
\hline \multicolumn{8}{|c|}{ 2006B South } \\
\hline ESO303-005 & 55 & - & 9.1 & 0.25 & 9.1 & 0.50 & 0.70 \\
\hline ESO 349-010 & 14 & 155 & 15.3 & 0.52 & 12.3 & 1.18 & 0.30 \\
\hline ESO 488-027 & 88 & 68 & 10.3 & 0.15 & 10.1 & 0.94 & 2.20 \\
\hline ESO 552-020 & 148 & 148 & 18.3 & 0.43 & 18.3 & 0.76 & 0.64 \\
\hline IC 1633 & 97 & 97 & 23.9 & 0.17 & 23.9 & 0.75 & 0.00 \\
\hline IC 5358 & 40 & 114 & 17.4 & 0.60 & 8.3 & 1.44 & 0.26 \\
\hline MCG-02-12-039 & 166 & 180 & 15.2 & 0.19 & 15.1 & 1.13 & 0.64 \\
\hline NGC 1399 & 222 & - & 42.2 & 0.06 & 42.2 & 0.44 & 0.45 \\
\hline NGC 7012 & 289 & 100 & 15.8 & 0.44 & 15.6 & 0.96 & 0.00 \\
\hline PGC 026269 & 313 & 133 & 7.3 & 0.00 & 7.3 & 0.89 & 0.00 \\
\hline \multicolumn{8}{|c|}{ 2006B North } \\
\hline NGC 1713 & 330 & 39 & 15.5 & 0.14 & 14.0 & 1.07 & 0.00 \\
\hline NGC 2832 & 226 & 172 & 21.2 & 0.17 & 19.6 & 1.12 & 0.50 \\
\hline NGC 7597 & 46 & 133 & 9.4 & 0.00 & 9.4 & 0.64 & 0.00 \\
\hline NGC 7649 & 78 & 78 & 12.8 & 0.31 & 12.8 & 0.82 & 0.20 \\
\hline UGC 02232 & 60 & - & 9.7 & 0.00 & 9.7 & 0.82 & 0.00 \\
\hline \multicolumn{8}{|c|}{ 2007A South } \\
\hline ESO 146-028 & 154 & 154 & 12.4 & 0.35 & 12.4 & 0.48 & 0.15 \\
\hline ESO 346-003 & 297 & 118 & 11.9 & 0.18 & 11.9 & 0.29 & 0.15 \\
\hline ESO 444-046 & 196 & 161 & 16.0 & 0.29 & 14.9 & 0.24 & 0.45 \\
\hline GSC555700266 & 204 & - & 10.6 & 0.30 & 10.6 & 0.47 & 0.00 \\
\hline IC 1101 & 204 & 25 & 12.2 & 0.50 & 12.2 & 0.39 & 0.20 \\
\hline IC 4765 & 287 & 123 & 28.0 & 0.46 & 27.1 & 0.13 & 0.75 \\
\hline LEDA094683 & 226 & 46 & 7.3 & 0.33 & 7.3 & 0.54 & 2.00 \\
\hline NGC 3311 & 63 & - & 26.6 & 0.17 & 26.6 & 0.53 & 0.40 \\
\hline NGC 4946 & 134 & 135 & 17.0 & 0.20 & 17.0 & 0.74 & 0.00 \\
\hline PGC 044257 & 267 & 44 & 15.3 & 0.63 & 10.5 & 0.38 & 0.00 \\
\hline PGC 072804 & 76 & 76 & 7.6 & 0.16 & 7.6 & 0.52 & 0.00 \\
\hline UGC 05515 & 293 & 83 & 12.0 & 0.13 & 11.8 & 0.38 & 0.00 \\
\hline \multicolumn{8}{|c|}{ 2007A North } \\
\hline NGC 4839 & 63 & 64 & 17.2 & 0.52 & 17.2 & 0.61 & 0.00 \\
\hline NGC 6086 & 270 & 3 & 11.2 & 0.29 & 8.1 & 0.51 & 0.10 \\
\hline NGC 6160 & 140 & 72 & 13.9 & 0.47 & 9.0 & 0.41 & 0.00 \\
\hline NGC 6173 & 139 & 138 & 15.0 & 0.26 & 15.0 & 0.47 & 0.10 \\
\hline NGC 6269 & 306 & 80 & 14.1 & 0.20 & 13.1 & 0.50 & 0.35 \\
\hline PGC 071807 & 138 & 138 & 6.7 & 0.70 & 6.7 & 0.71 & 0.10 \\
\hline
\end{tabular}

images taken directly before and/or after the science frames (i.e. at the same telescope pointing). It was necessary to make an illumination correction using normalized twilight flat-field spectra to correct for spatial gradients in the star and galaxy spectra. Full twodimensional wavelength calibration was performed and star and galaxy spectra were transformed to equal linear spectral bin sizes.

All the flux calibration and Lick stars were sky-subtracted and $S$-distortion-corrected before one-dimensional spectra were extracted. All one-dimensional Lick star spectra were flux calibrated and corrected for atmospheric extinction.

A cosmic ray cleaning algorithm was applied to all the twodimensional galaxy spectra before they were background-subtracted and $S$-distortion-corrected. For each individual galaxy frame, a sky region consisting of 40 rows was chosen on each side of the galaxy and averaged together to obtain a one-dimensional sky spectrum. The sky spectrum was grown into a two-dimensional spectrum and subtracted from the data frame. Flux calibration and extinction corrections were also applied to the two-dimensional spectra. The galaxy frames were then co-added (also removing any remaining cosmic ray effects in the process) to form a single frame for each galaxy in each of the blue and red arms and for each of the two dichroics.

The basic Gemini data reductions were performed with the Gemini-specific GMOS data reduction package that can be added on to the standard IRAF reduction package. Spectra with different central wavelengths (5080 and $5120 \AA$ ) and spectra taken with GMOS-North and GMOS-South were treated in similar parallel streams of data reduction. The raw Lick star data were obtained from the Gemini Science Archive. ${ }^{6}$ The basic data reductions were

\footnotetext{
${ }^{6}$ http://www4.cadc-ccda.hia-iha.nrc-cnrc.gc.ca/gsa/
} 
conducted in a similar manner as for the WHT data. For each of the two central wavelength settings, a bad pixel spectrum was made in order to avoid interpolation between the gaps. When the spectra of the two different wavelength settings were combined, these bad pixel masks were used to ensure that only true data were averaged.

Proctor et al. (2005) and Brough et al. (2007) also used GMOS data to measure Lick indices, and found negligible scattered light that influenced their results. Nevertheless, as a test of the effect of scattered light in the GMOS spectrograph on our data, bright skylines were used to extract flat-field profiles that could be compared to the shape of the dome flats that were used. It was found that scattered light effects did not influence the regions where galaxy data were extracted or the regions used for background subtraction.

An error analysis was completed to account for both Poisson (photonic) noise and systematic errors in the data. Variance frames were created from the data frames after bias subtraction and ADU conversion, and reduced in a way similar to the data frames, but the arithmetic manipulations were performed by incorporating the usual error propagation laws. Thus, the variance frames represent the square of the Poisson noise at the start of this reduction process, and all other systematic noise effects are added on to the variance frames in quadrature.

Flat-fielding produced negligible errors with the exception of the WHT flat-field spectra taken in the upper wavelength region (red spectra of dichroic $6100 \AA$ on night four), which contained fringe patterns. This contributed about 4 per cent to the errors, and these were appropriately propagated in the variance frame. Errors contributed by the illumination correction proved negligible. Wavelength calibration was accurate to within $0.1 \AA$ in all cases. After that, the variance frames were also $S$-distortion-corrected, following the procedure used for the corresponding data frames. The sky subtraction process added to the uncertainty in the data and was properly taken into account by calculating the following for each variance frame:

$[\Delta \operatorname{Sky}(\lambda)]^{2}=\left[\frac{1}{\sqrt{N}} \Delta A(\lambda)\right]^{2}$,

where $\Delta$ Sky is the error contribution of the sky subtraction being taken into account, $N$ is the number of spatial cross-sections averaged for the sky determination and $A$ is the Poisson error on the average one-line sky spectrum. This one-dimensional spectrum was then grown into a two-dimensional spectrum with the proper dimensions and added on to the variance frames in all cases.

Relative flux calibration was accurate to within 5 to 10 per cent, and the variance frames were also co-added so that they could be treated alongside the galaxy data in further analysis. For the Gemini data, variance frames were co-added for each galaxy by dealing with the gaps in the same manner as the data (described above) to avoid any interpolation. The fact that only $N / 2$ frames were added in the regions where the gaps occur, instead of $N$ individual frames per galaxy in all the other regions, was taken into account in the variance frame combination procedure. At the end of this reduction process, each two-dimensional galaxy data frame had an associated two-dimensional variance array.

As mentioned, some of the galaxies were observed at a position angle (PA) other than the MA, due to the two control elliptical galaxies in the WHT observations and the availability of suitably bright guide stars in the Gemini observations. This had an effect on the relative half-light radii $\left(r_{\mathrm{e}}\right)$, which had to be taken into account. Tables 2 and 3 list the $r_{\mathrm{e}}$ along the semimajor axis of the galaxies, computed from the Two-Micron All Sky Survey (2MASS) $K$-band 20th magnitude $\operatorname{arcsec}^{-2}$ isophotal radius using the formula by
Jarrett et al. (2003): $\log r_{\mathrm{e}} \sim \log r_{\mathrm{K} 20}-0.4$. Using the ellipticities $(\epsilon)$ of the galaxies (data from NED), the effective half-light radii at PA were computed according to

$a_{\mathrm{e}}=\frac{r_{\mathrm{e}}(1-\epsilon)}{1-\epsilon|\cos (|\mathrm{PA}-\mathrm{MA}|)|}$.

The fraction of $a_{\mathrm{e}}$ which the radial profiles measured in this work spans is also listed in Tables 2 and 3. For those galaxies for which a MA angle is not available in HyperLeda, the PA is assumed to be the same as the MA (as intended during the observation preparations).

\section{CENTRAL KINEMATICAL MEASUREMENTS}

The central radial velocity and velocity dispersion values of the galaxies were derived for regions with the size of $a_{\mathrm{e}} / 8$, and with the galaxy centres defined as the luminosity peaks. Thus, the central values are for an aperture of $1 \times a_{\mathrm{e}} / 8 \mathrm{arcsec}^{2}$ for the WHT data and $0.5 \times a_{\mathrm{e}} / 8 \operatorname{arcsec}^{2}$ for the Gemini data.

The Lick stars were shifted to zero radial velocity before using them as templates. Radial velocities and velocity dispersions of the galaxies were measured using the MOVEL and OPTEMA algorithms described by González (1993) and integrated into the REDUCEME data reduction package. ${ }^{7}$ This routine is an improvement of the classic Fourier Quotient Technique described by Sargent et al. (1977). It is an iterative procedure in which a galaxy model is processed in parallel with the galaxy spectrum. In this way, a comparison between the input and recovered broadening functions for the model allows one to correct the galaxy power spectrum for any imperfections of the data handling procedures in Fourier Space. The procedure uses an algorithm that obtains the linear combination of individual templates that best matches the observed galaxy spectrum. The best template is scaled, shifted and broadened to match the galaxy kinematic parameters and is feeded to the MOVEL algorithm. The output kinematic parameters are then used to create and improve composite templates, and the process continues until it converges. This OPTEMA algorithm is able to overcome the typical template mismatch problem (see van der Marel \& Franx 1993) by constructing an optimal template as a linear combination of stellar spectra of different spectral types and luminosity classes for each galaxy (González 1993; Sánchez-Blázquez et al. 2006). A new optimal template was constructed with each spatial bin in the kinematical measurements.

Skyline residuals and emission lines were masked. Emission lines were detected in 13 galaxies: ESO 349-010, MCG-02-12-039, NGC 0541, NGC 1713, NGC 3311, NGC 4874, NGC 4946, NGC 6166, NGC 6173, NGC 7012, NGC 7649, NGC 7720 and PGC 044257. The detection thresholds and emission-line correction will be described in a future paper devoted to the stellar populations.

For the Gemini data, 20 stars were used to make the optimal template (excluding the stars not of $G$ or $K$ spectral type), and 22 stars for the WHT data. Fig. 1 shows a typical fit between the observed central spectrum of a galaxy and the optimal template for that galaxy corrected by the derived kinematic parameters. ${ }^{8}$

\footnotetext{
${ }^{7}$ REDUCEME is an astronomical data reduction package, specially devoted to the analysis of the long-slit spectroscopy data. It was developed by N. Cardiel and J. Gorgas (Cardiel 1999). http://www.ucm. es/info/Astrof/software/reduceme/reduceme.html

${ }^{8}$ The derived recession velocities were automatically corrected for the standard special relativistic correction $z=\left(1+\frac{V_{\mathrm{nr}}}{c}\right) \gamma-1$, where $\gamma=$ $\frac{c}{\sqrt{c^{2}-V^{2}}}, V_{\mathrm{nr}}$ is the non-relativistic radial velocity and $c$ is the speed of light.
} 


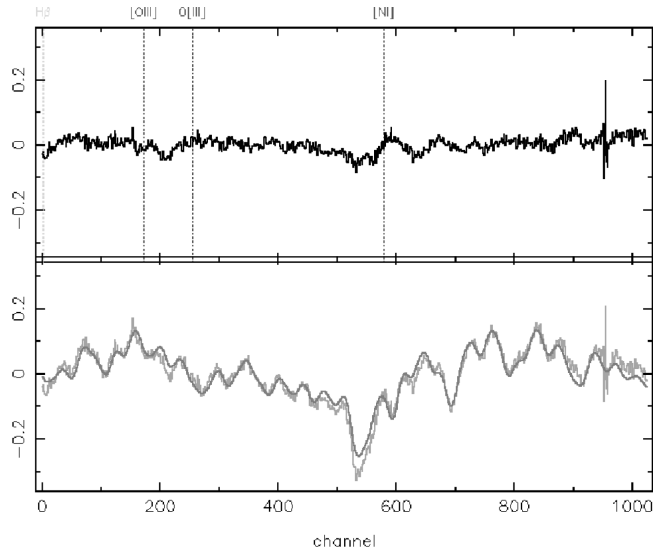

Figure 1. A typical example of the calculation of the derived kinematics for NGC 7768 with the MOvEL algorithm. In the lower panel, the smooth line corresponds to the optimal template spectrum, plotted together with the galaxy spectrum which has been shifted and broadened to the derived parameters. The upper panel shows the residuals of fit (in relative flux units), with the dotted lines indicating the positions of typical emission lines. This illustration spans 4900 to $5400 \AA$.

The errors were computed through Monte Carlo numerical simulations and using the appropriate error spectra. In each simulation, Gaussian noise was added to the galaxy spectra in accordance with the error spectra and the radial velocity and velocity dispersion derived. Errors in the radial velocities and velocity dispersions were calculated as the unbiased standard deviation of the different solutions. The errors computed in this manner are expected to incorporate all the uncertainties, from the basic reduction process (which is accounted for in the error spectra) to the final measurement of the parameters.

When comparing measured central velocity dispersion values with those from the literature, emphasis must be placed on the fact that published values are measurements from within different apertures, which can have a systematic effect on the values. Accurate comparison is further limited because of different effective spatial resolution, data of different quality and different analysis techniques.

Fig. 2 shows the rough comparison (not taking into account the above differences) between the velocity dispersion values from HyperLeda (if available) and those derived in this study. For most of the galaxies, more than one velocity dispersion values are listed in HyperLeda (in which case the most recent was used for this comparison). In the case of NGC 1399, for example, published values range between 233 and $420 \mathrm{~km} \mathrm{~s}^{-1}$, which shows the effect different apertures and measurements have (this can be seen in the steep change in the measured velocity dispersion profile of NGC 1399 in Fig. 4). The absolute mean difference between the values measured here and the literature is $\mid \sigma$ (here) $-\sigma$ (lit, uncorrected) $\mid=33 \pm$ $29 \mathrm{~km} \mathrm{~s}^{-1}$, which is acceptable for the purposes of this paper.

\section{KINEMATICS OF INDIVIDUAL GALAXIES}

The galaxy spectra were binned in the spatial direction to ensure a minimum signal-to-noise ratio of 20 per $\AA$ in the $\mathrm{H} \beta$ region of the spectrum for kinematical measurements as a function of radius. Thus, the spatial cross-sections are broader with increasing radius from the centre of the galaxy. The radial kinematics (radial velocities and velocity dispersions) were measured with the same MOVEL and OPTEMA algorithms in the REDUCEME package and with the same

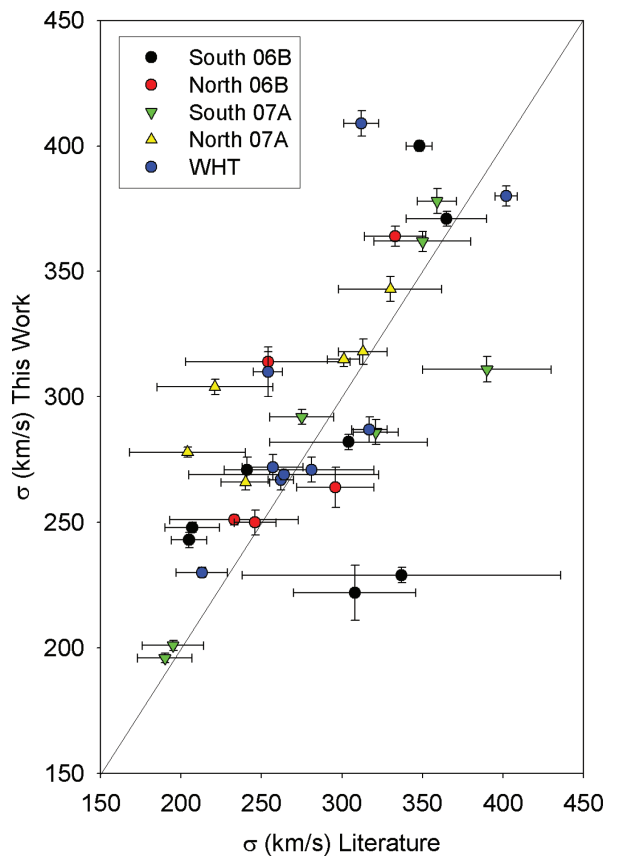

Figure 2. Velocity dispersion results compared to literature values. The latter values are the most recent ones from HyperLeda as described in the text.

optimal Lick star templates as the central kinematics. The values of the kinematic parameters are evaluated at the luminosity-weighted centres of the spatial bins used to derive the parameters.

Dark time at both Gemini telescopes is highly oversubscribed. To speed up the completion of this multi-semester project, we relaxed the observing conditions to accept grey time as well. However, as a consequence, it was not possible to extract radial profiles to very large radii for those targets observed during grey time, but the central values are still accurate. Eight galaxies (three WHT and five Gemini) could be extracted to radii $>1 a_{\mathrm{e}}$ and 12 galaxies (one WHT and 11 Gemini) could only be extracted to radii $<0.5 a_{\mathrm{e}}$. The rotational velocity $V_{\max }$ was measured as half the difference between the peaks of the rotation curve.

\subsection{Notes on individual objects}

This section describes the individual objects and their radial kinematic profiles as shown in Figs 3-7. We refer to results published prior to this study, as well as the results obtained here. The radial kinematic profiles of NGC 4839, NGC 4889, NGC 6166 and IC 1101 are compared to previous measurements from the literature in Appendix A. Appendix B contains the radial kinematics of the individual galaxies in tabular form, which is available in the online version of this article.

ESO 146-028: the radial velocity of this galaxy is mostly flat and the velocity dispersion profile is decreasing outwards.

ESO 303-005: the radial kinematic profiles of this galaxy show no significant rotation or substructure.

ESO 346-003: the radial velocity profile shows clear rotation of the order of $51 \mathrm{~km} \mathrm{~s}^{-1}$.

ESO 349-010: HST imaging of this galaxy was published by Laine et al. (2003). The radial velocity profile of this galaxy shows 


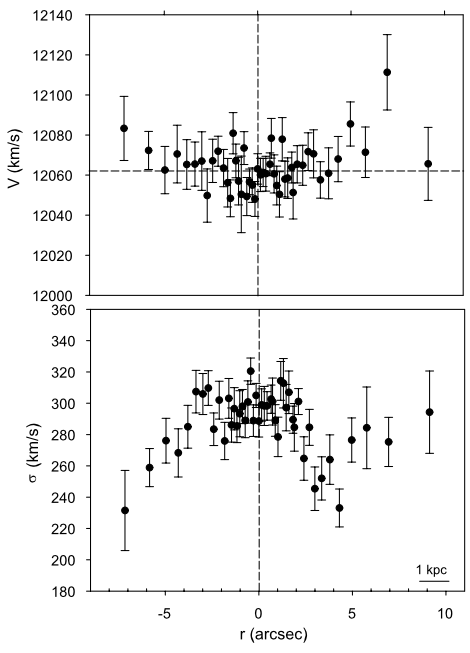

(a) ESO146-028.

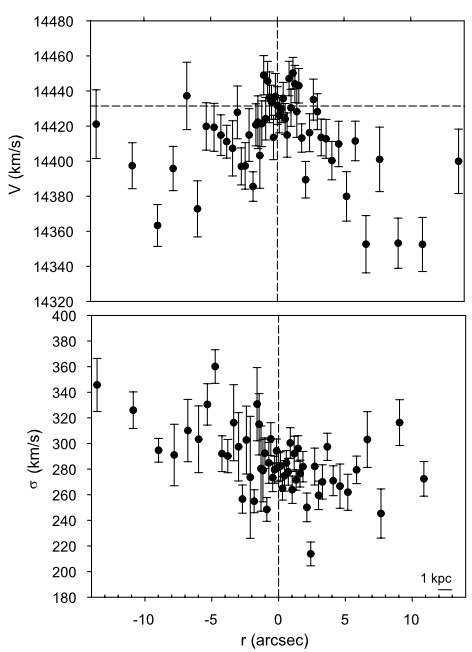

(d) ESO349-010.

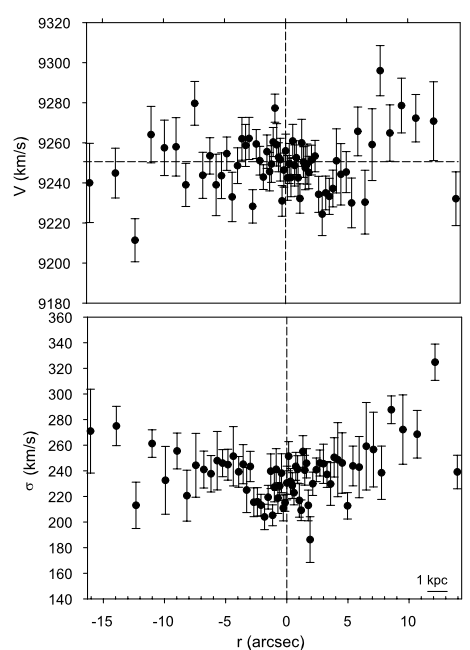

(g) ESO552-020

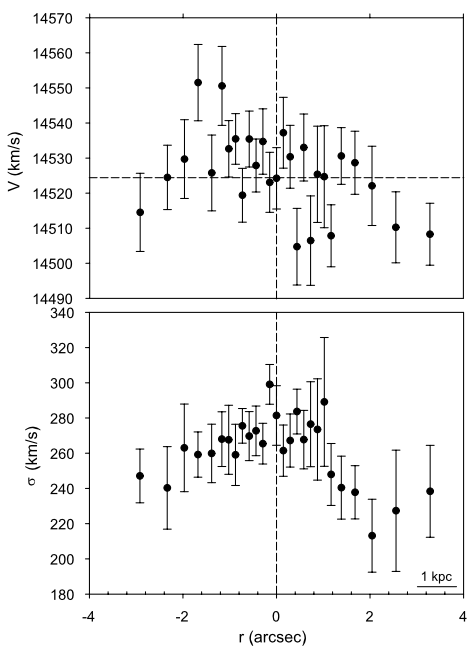

(b) ESO303-005

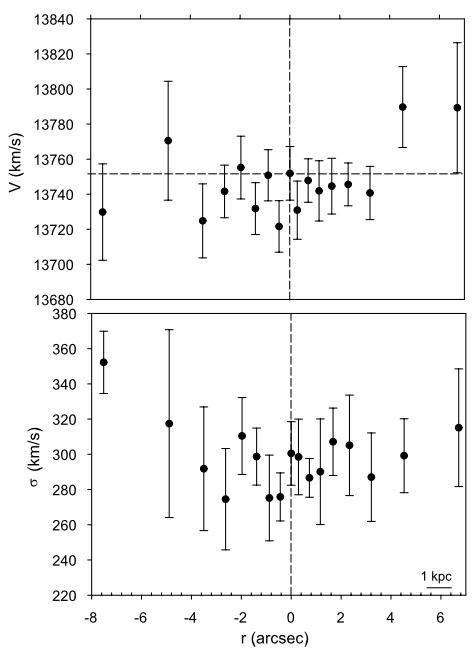

(e) ESO444-046

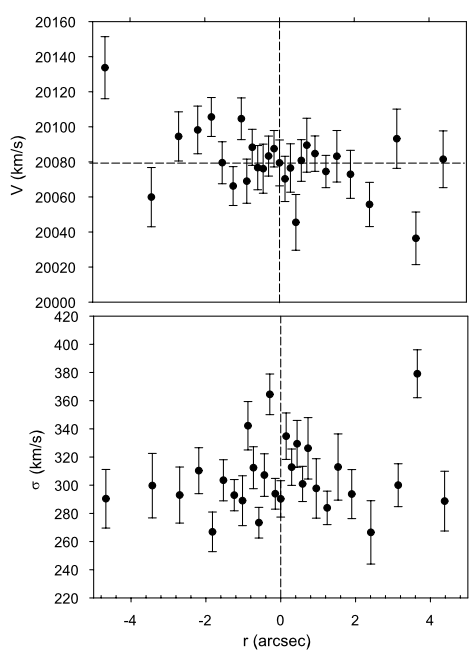

(h) GSC555700266

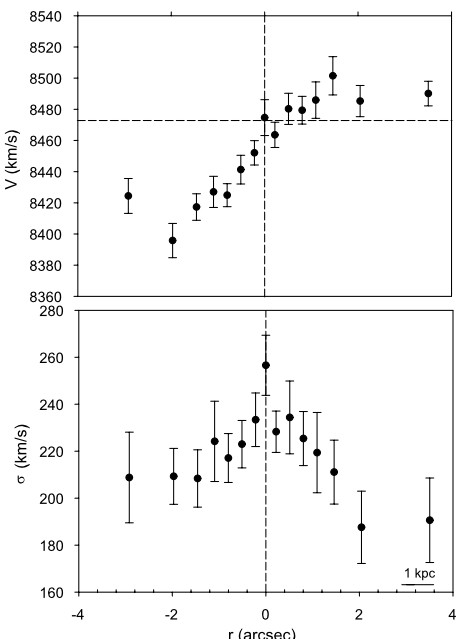

(c) ESO346-003.

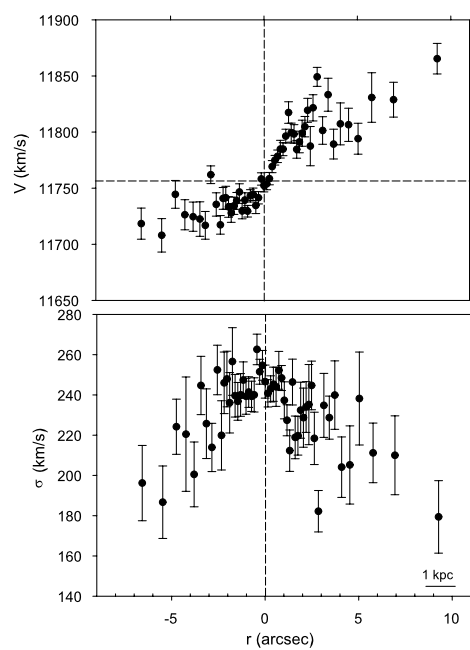

(f) ESO488-027.

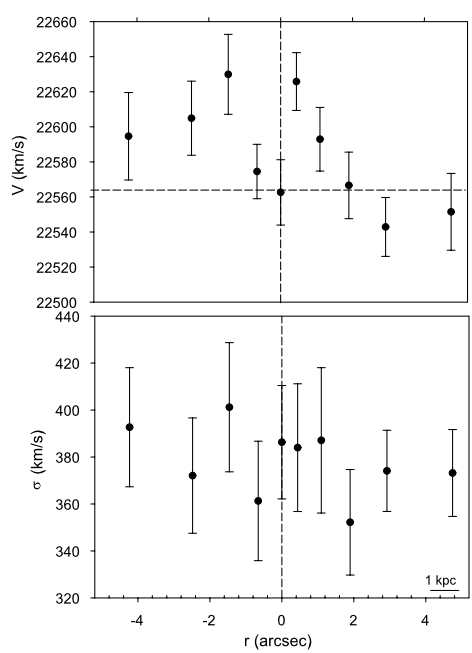

(i) IC1101

Figure 3. Radial profiles of velocities $(V)$ and velocity dispersion $(\sigma)$. The vertical lines indicate the centre of the galaxy and the horizontal line the radial velocity of the central bin. 


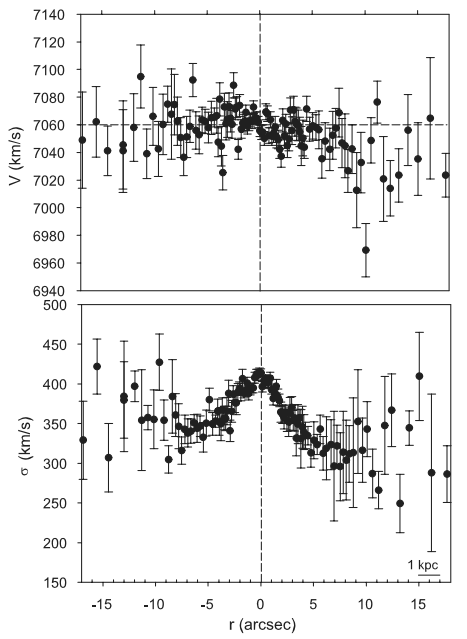

(a) IC1633.

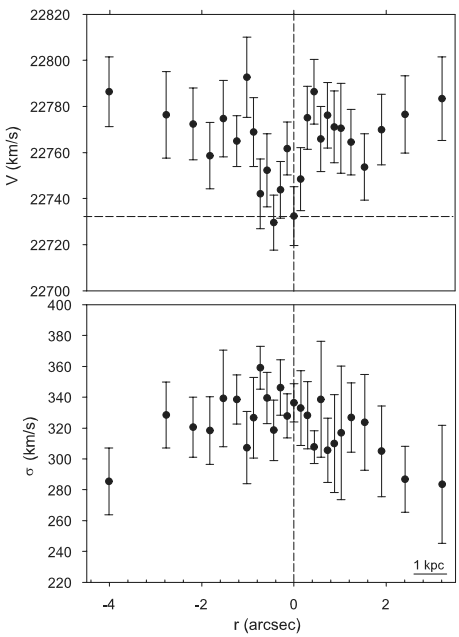

(d) Leda094683.

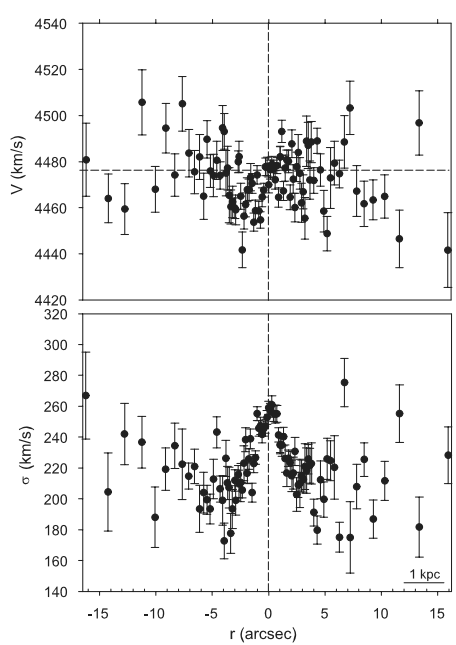

(g) NGC1713

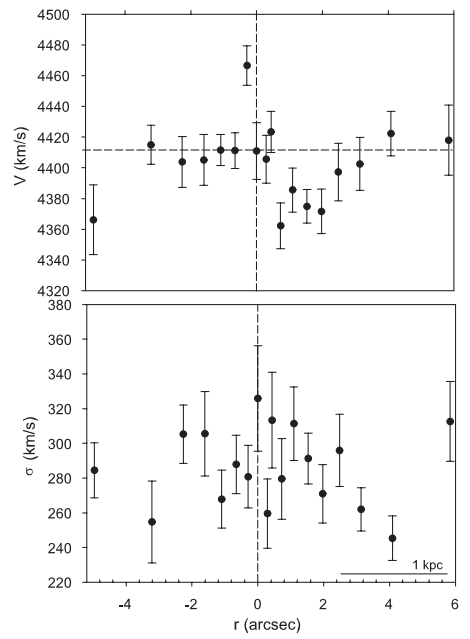

(b) IC4765.

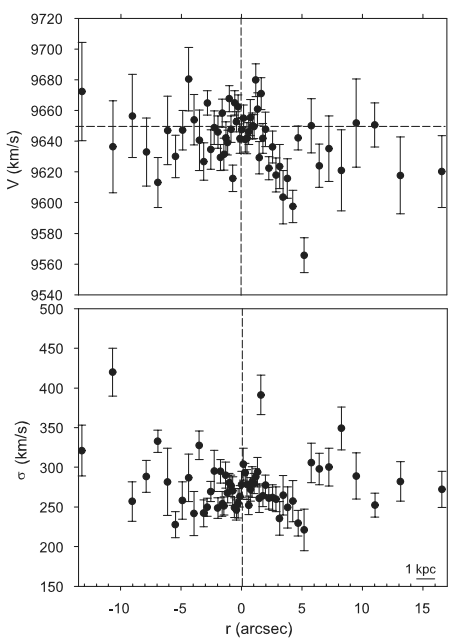

(e) MCG-02-12-039.

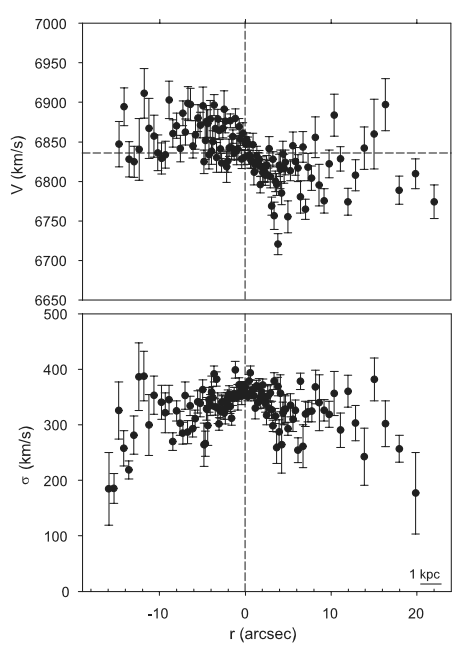

(h) NGC2832

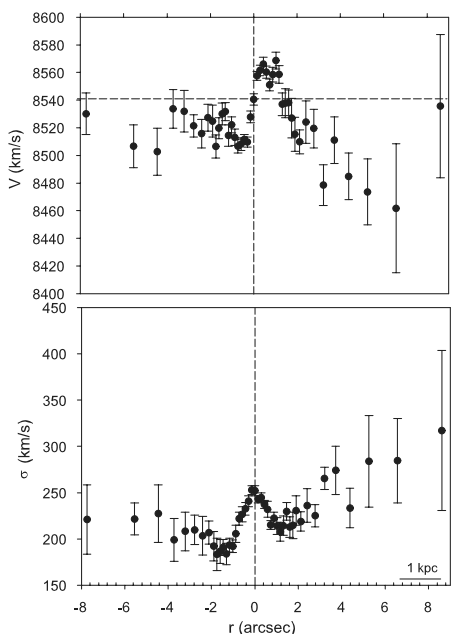

(c) IC5358.

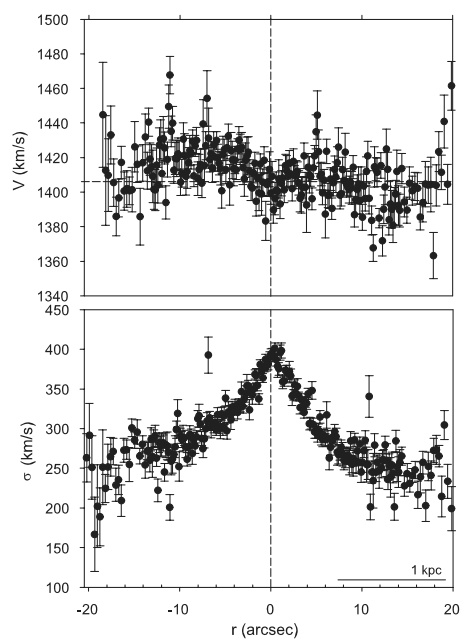

(f) NGC1399.

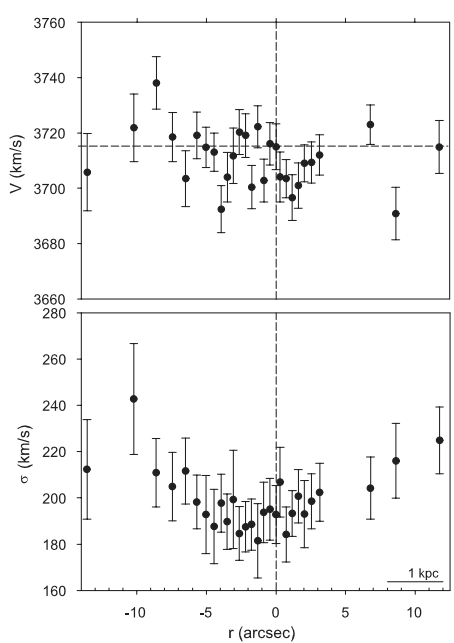

(i) NGC3311

Figure 4. Radial profiles of velocities $(V)$ and velocity dispersion $(\sigma)$. The dashed lines are as in Fig. 3 . 


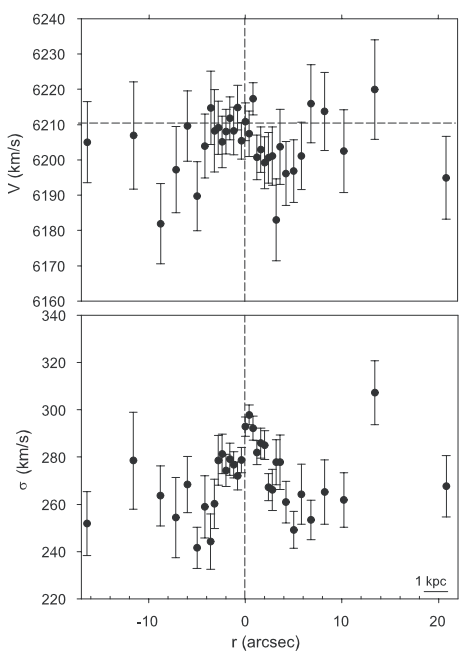

(a) NGC3842.

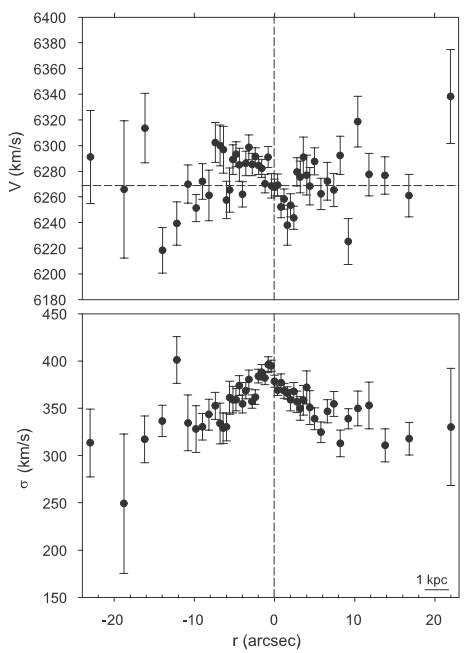

(d) NGC4889.

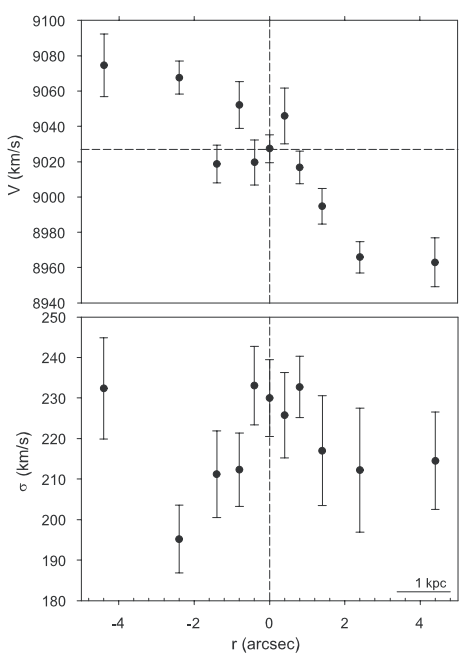

(g) NGC6047

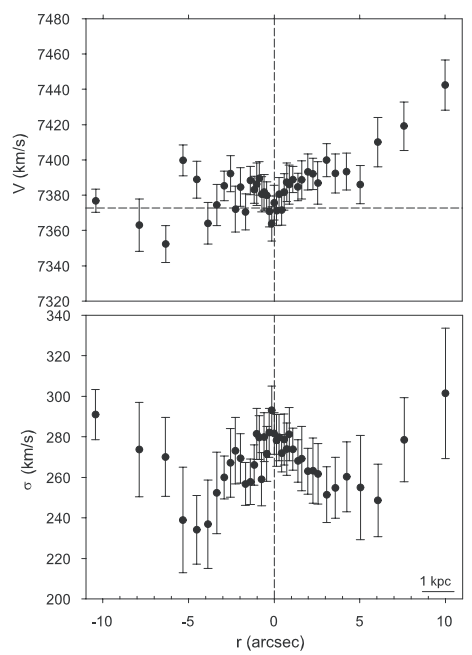

(b) NGC4839.

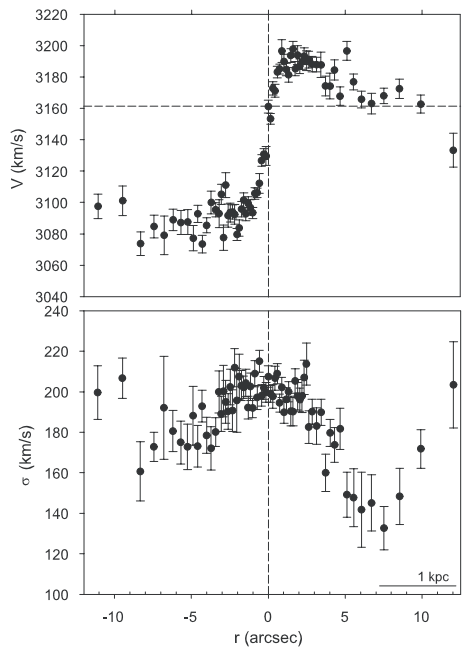

(e) NGC4946.

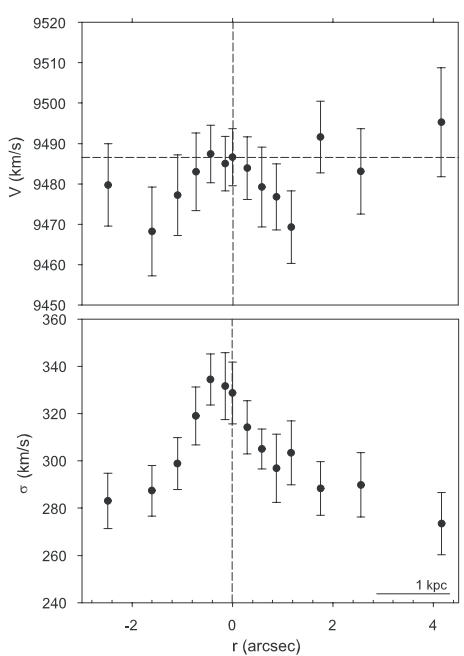

(h) NGC6086

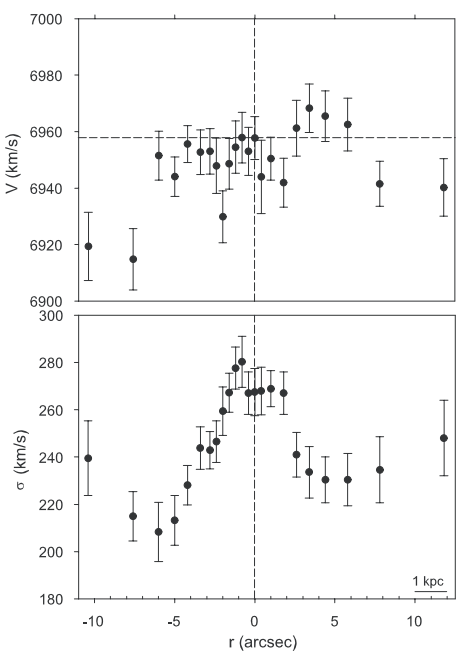

(c) NGC4874.

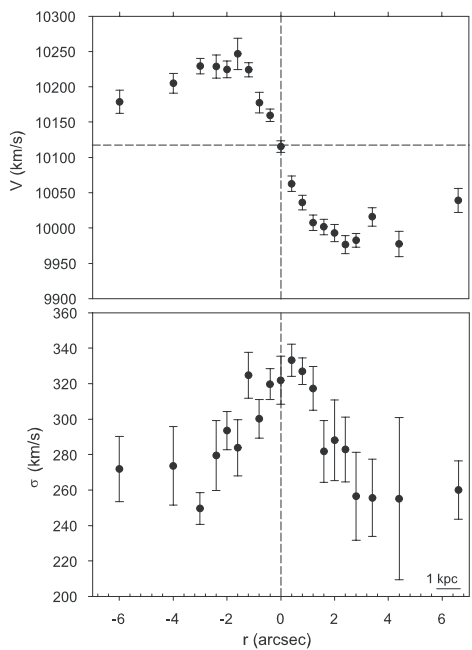

(f) NGC6034.

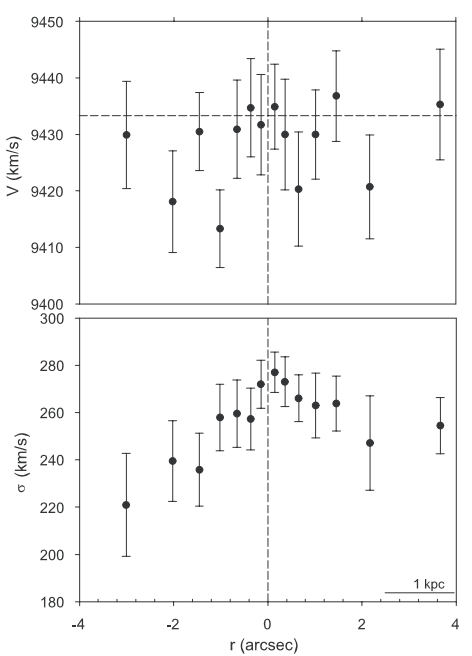

(i) NGC6160

Figure 5. Radial profiles of velocities $(V)$ and velocity dispersion $(\sigma)$. The dashed lines are as in Fig. 3 . 


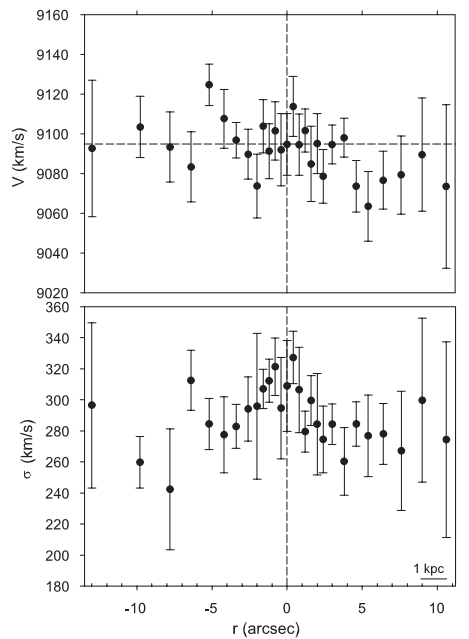

(a) NGC6166.

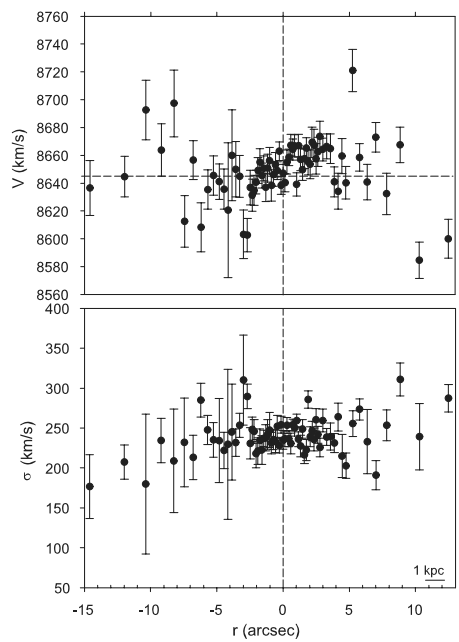

(d) NGC7012.

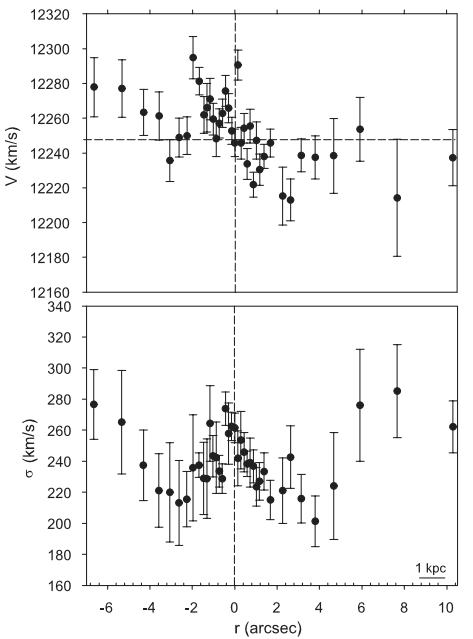

(g) NGC7649

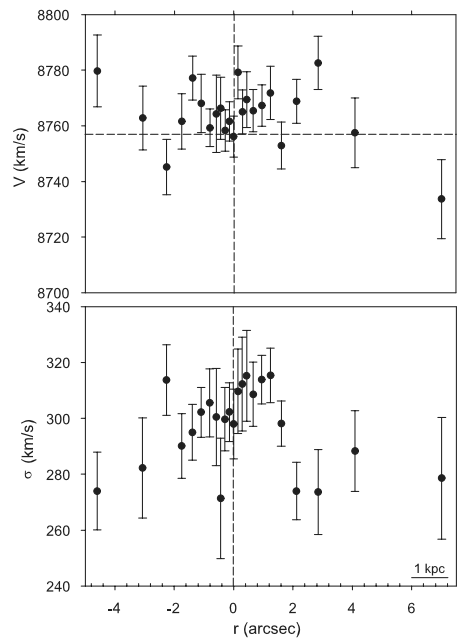

(b) NGC6173.

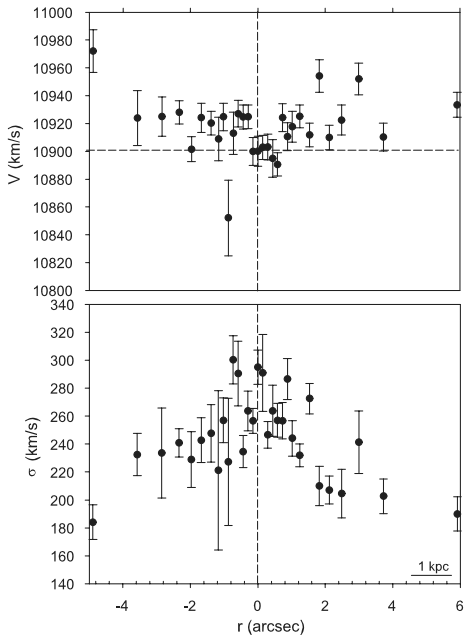

(e) NGC7597.

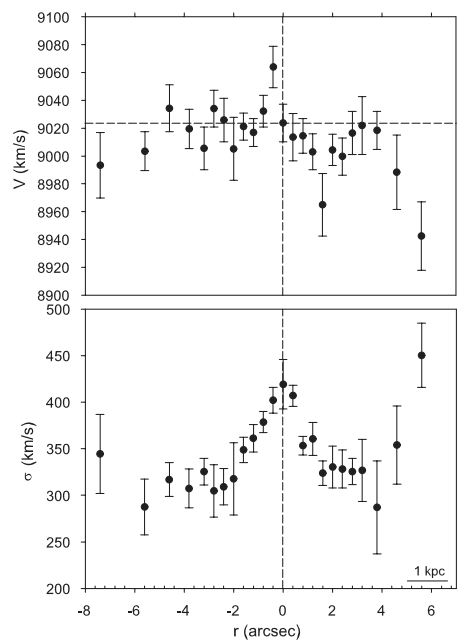

(h) NGC7720

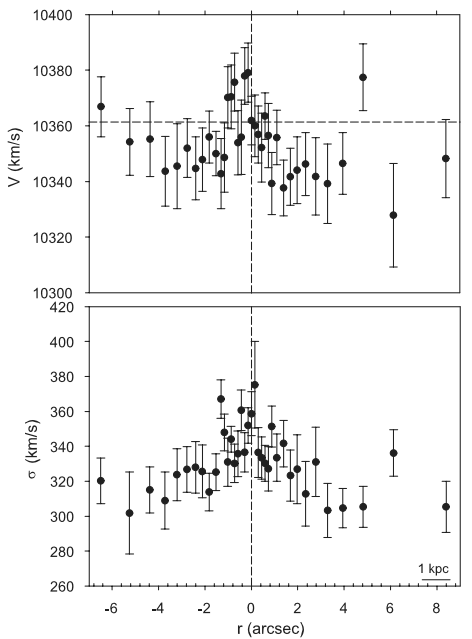

(c) NGC6269.

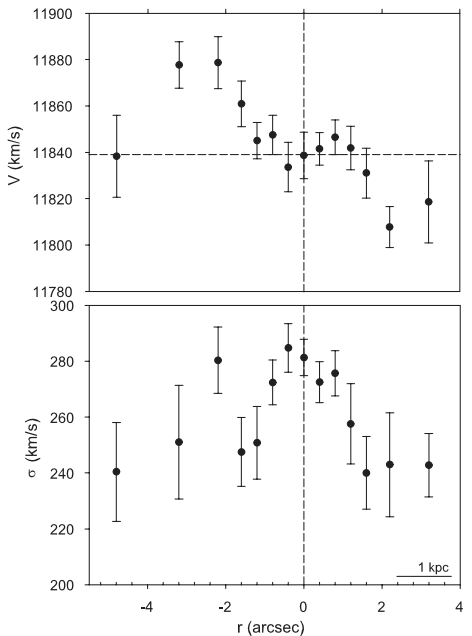

(f) NGC7647.

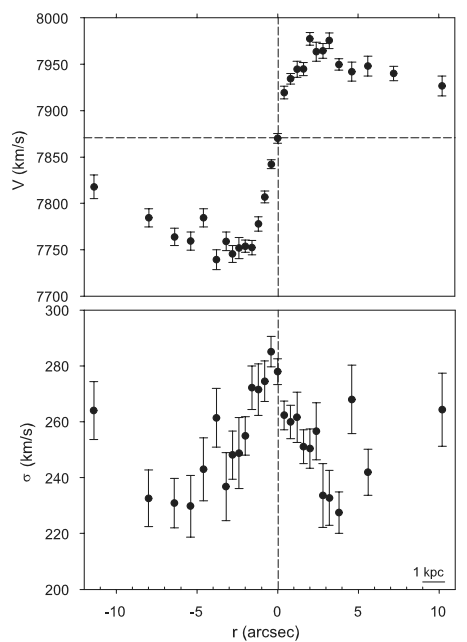

(i) NGC7768

Figure 6. Radial profiles of velocities $(V)$ and velocity dispersion $(\sigma)$. The dashed lines are as in Fig. 3 . 


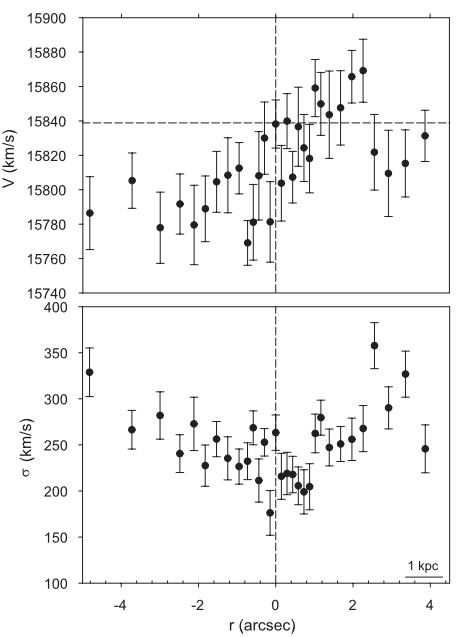

(a) PGC026269.

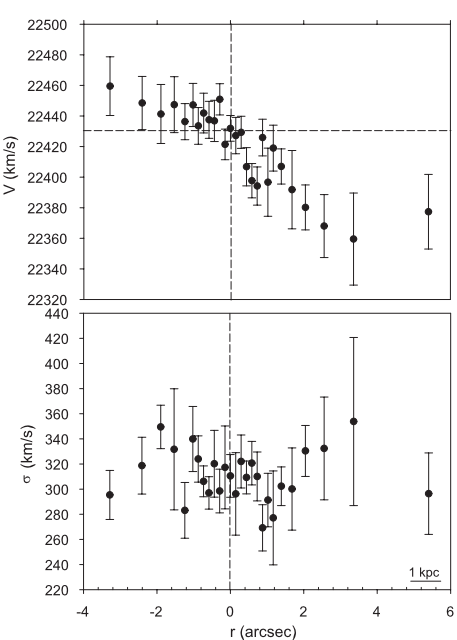

(d) PGC072804.

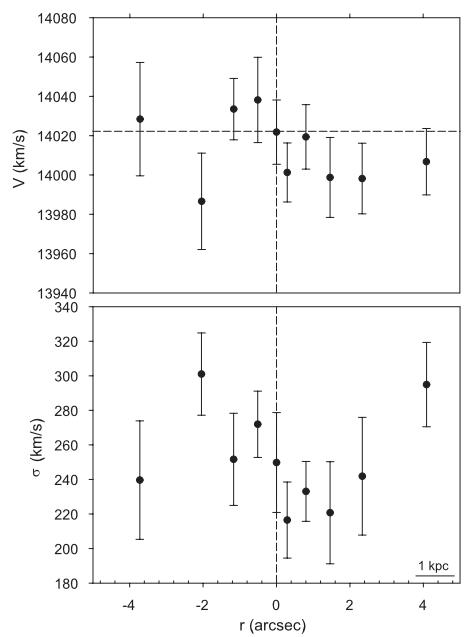

(b) PGC044257.

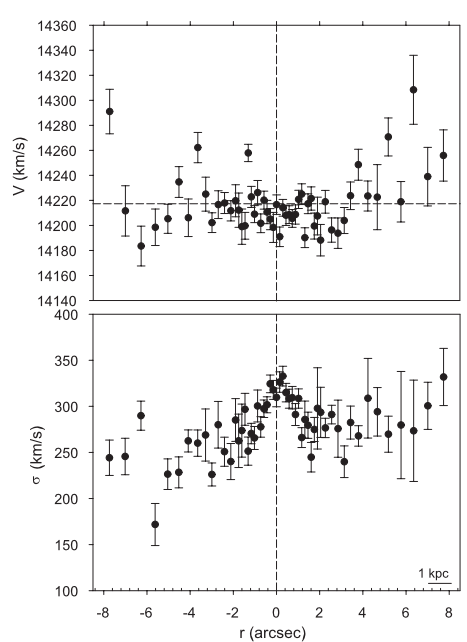

(e) UGC02232.

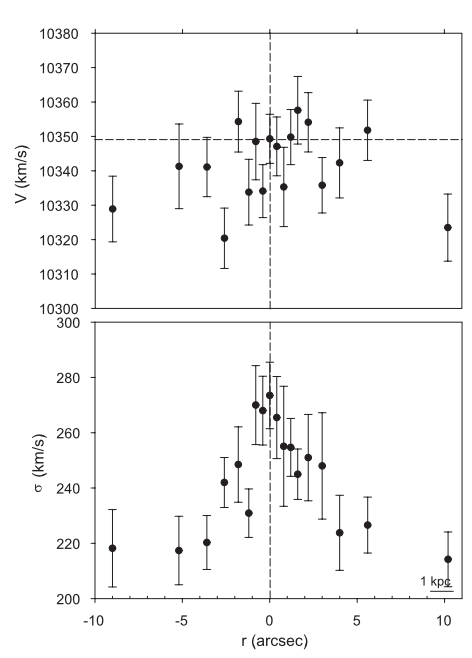

(g) UGC10143

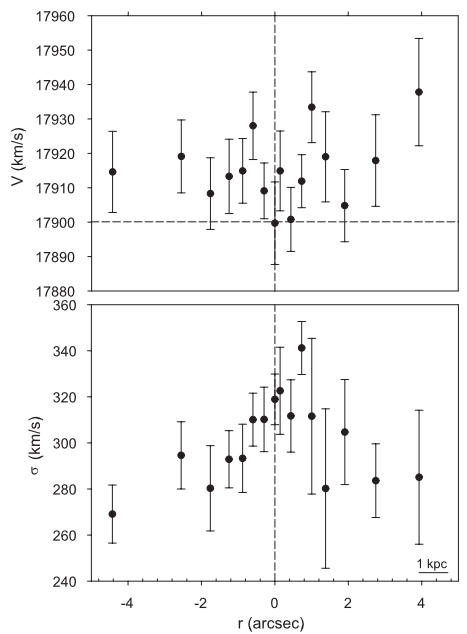

(c) PGC071807.

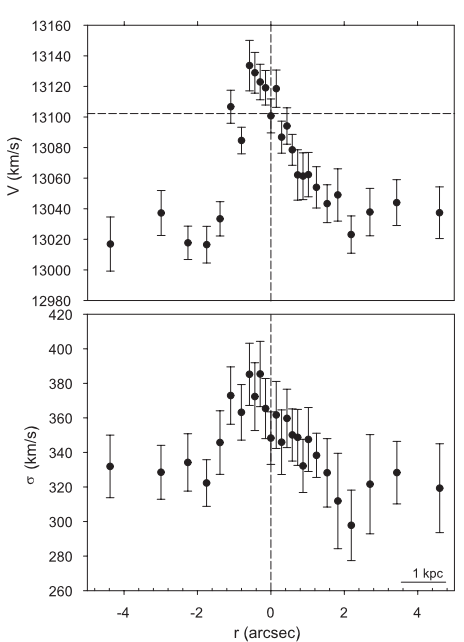

(f) UGC05515.

(g) UGC10143

Figure 7. Radial profiles of velocities $(V)$ and velocity dispersion $(\sigma)$. The dashed lines are as in Fig. 3. 
no significant rotation, and the velocity dispersion profile has a positive gradient.

ESO 444-046: no clear rotation or velocity substructure is visible in the kinematic profiles.

ESO 488-027: the kinematic profiles show a kinematically decoupled core (KDC) in the centre of this galaxy.

ESO 552-020: the radial velocity profile is mostly flat and the velocity dispersion profile shows a small dip south-west of the centre of the galaxy, which might indicate a colder component.

GSC555700266: slow rotation but no clear substructure can be seen in the kinematic profiles.

IC 1101: this galaxy is one of the few known BCGs that were previously found to have a rising velocity dispersion profile (Fisher et al. 1995a). This result cannot be confirmed here since the derived velocity dispersion profile is not measured to the same radius as in the above-mentioned study. No significant rotation is detected in the radial velocity profile.

IC 1633: the surface brightness profile was published by Schombert (1986) and HST imaging by Laine et al. (2003). The radial velocity profile reveals some evidence for substructure at the centre of this galaxy.

IC 4765: the radial velocity profile shows rotation in the centre of the galaxy, which is possibly rotational substructure.

IC 5358: both the radial kinematic profiles show the clear presence of a KDC in this galaxy.

LEDA094683: no significant rotation but some evidence of substructure is visible from the radial velocity profile.

MCG-02-12-039: this BCG is a weak line emitter. The surface brightness profile was published by Schombert (1986) and HST imaging by Laine et al. (2003). A KDC is detected in the radial kinematic profiles.

NGC 1399: the surface brightness profile was published by Schombert (1986). The velocity dispersion in the central region of this galaxy was found to be $388 \mathrm{~km} \mathrm{~s}^{-1}$ decreasing to $200 \mathrm{~km} \mathrm{~s}^{-1}$ at 10 arcsec from the centre by Longo et al. (1994). The central radial velocity dispersion of this galaxy was measured in this study as $371 \pm 3 \mathrm{~km} \mathrm{~s}^{-1}$. Thus, the central velocity dispersion measured in this study corresponds to that measured by Longo et al. (1994), and the steep decreasing velocity dispersion profile is confirmed as well. This galaxy has a flat radial velocity profile.

NGC 1713: the radial velocity profile of this galaxy reveals a definite KDC in the centre of this galaxy.

NGC 2832: this galaxy is thought to be tidally interacting with its companion, NGC 2831. The surface brightness profile was published by Schombert (1986) and Jordán (2004) and the HST imaging by Laine et al. (2003). The radial velocity profile shows the presence of a KDC in the centre of this galaxy.

NGC 3311: the HST imaging was published by Laine et al. (2003). No clear rotation or velocity substructure can be seen in the radial kinematic profiles, but the velocity dispersion profile has a positive gradient.

NGC 3842: this galaxy belongs to a cluster, which forms a part of the Coma supercluster. The surface brightness profile was published by Schombert (1986) and the HST imaging by Laine et al. (2003). The radial velocity profile of this galaxy reveals the presence of a $\mathrm{KDC}$ in the centre.

NGC 4839: the location of this cD galaxy far from the centre of the Coma cluster is unusual. Surface brightness profiles were published by Schombert (1986), Oemler (1976) as well as Jordán (2004), who all confirm the presence of a very prominent $\mathrm{cD}$ envelope. Rotation of the order of $44 \mathrm{~km} \mathrm{~s}^{-1}$ and a KDC in the centre of the galaxy are detected.
NGC 4874: this galaxy has a large, extended envelope. It is the second brightest galaxy of the famous pair of cDs at the centre of the Coma cluster. The surface brightness profile was published by Peletier et al. (1990). No significant rotation or velocity substructure is detected for this galaxy.

NGC 4889: a large cD galaxy with a very extended envelope. This is the brightest galaxy of the Coma cluster. The surface brightness profile was published by Peletier et al. (1990) and the HST imaging by Laine et al. (2003). The radial velocity profile clearly shows a KDC.

NGC 4946: this is an ordinary elliptical galaxy. The radial velocity profile of this galaxy reveals rotation of the order of $62 \mathrm{~km} \mathrm{~s}^{-1}$ along the MA.

NGC 6034: the surface brightness profile was published by Schombert (1986). The radial velocity profile of this galaxy shows a significant rotation of the order of $134 \pm 15 \mathrm{~km} \mathrm{~s}^{-1}$ along the MA.

NGC 6047: the surface brightness profile was published by Schombert (1986), and Schombert (1987) classified it as an E/SO galaxy. The radial velocity profile of this galaxy shows rotation of the order of $59 \mathrm{~km} \mathrm{~s}^{-1}$ (with the slit placement $29^{\circ}$ from the MA).

NGC 6086: the surface brightness profile was published by Schombert (1986) and the HST imaging by Laine et al. (2003). Carter et al. (1999) found this galaxy to have a KDC. Even though some evidence of substructure can be seen, this core cannot be confirmed since the profiles measured here do not cover the same radial extend as those in Carter et al. (1999).

NGC 6160: the radial velocity profile of this galaxy reveals no significant rotation or substructure.

NGC 6166: this is a classic multiple nucleus cD galaxy in a rich cluster. The surface brightness profile was published by Schombert (1986). Carter et al. (1999) found the velocity dispersion of this galaxy to increase from $325 \mathrm{~km} \mathrm{~s}^{-1}$ at the centre to $450 \mathrm{~km} \mathrm{~s}^{-1}$ at 35 arcsec along the MA. They also found the galaxy to show modest MA rotation $\left(45 \mathrm{~km} \mathrm{~s}^{-1}\right.$ at 40 arcsec). The profiles derived here do not extend out to the radii necessary to confirm the positive velocity dispersion slope found by Carter et al. (1999). Rotation of the order of $31 \mathrm{~km} \mathrm{~s}^{-1}$ is found in the centre of the galaxy, although this is not a very clear rotation curve.

NGC 6173: the surface brightness profile was published by Schombert (1986) and the HST imaging by Laine et al. (2003). The derived radial kinematic profiles of this galaxy show no significant rotation or substructure.

NGC 6269: surface brightness profiles were published by Schombert (1986) and by Malumuth \& Kirshner (1985). The radial velocity profile shows some velocity substructure in the centre of this galaxy. This galaxy was observed at an angle of $46^{\circ}$ away from the MA.

NGC 7012: the radial velocity profile shows a small amount of rotation in the centre which might be a KDC.

NGC 7597: the surface brightness profile was published by Schombert (1986). The radial kinematic profiles show possible evidence for a KDC.

NGC 7647: this is a cD galaxy with a red envelope and a large number of small companions (Vitores et al. 1996). The surface brightness profile was published by Schombert (1986) and the HST imaging by Laine et al. (2003). The radial velocity profile of this galaxy shows a clear KDC in the centre.

NGC 7649: the surface brightness profile was published by Schombert (1986) and the HST imaging by Laine et al. (2003). A KDC is clearly visible in the centre of this galaxy.

NGC 7720: this is a multiple nuclei galaxy and has a massive close companion galaxy, also an elliptical. The surface brightness 
profile was published by Lauer (1988) and the HST imaging by Laine et al. (2003). The radial velocity profile reveals no significant rotation but there might be some substructure in this galaxy.

NGC 7768: surface brightness profiles were published by Schombert (1986), Malumuth \& Kirshner (1985) and Jordán (2004) and the HST imaging was published by Laine et al. (2003). The radial velocity profile of this galaxy shows significant rotation of the order of $114 \pm 11 \mathrm{~km} \mathrm{~s}^{-1}$ along the MA. This agrees with the $101 \pm$ 5 and the $99 \mathrm{~km} \mathrm{~s}^{-1}$ rotations found previously by Fisher et al. (1995a) and Prugniel \& Simien (1996), respectively.

PGC 026269: also known as Hydra A. The radial kinematic profiles of this galaxy reveal rotation of the order of $51 \mathrm{~km} \mathrm{~s}^{-1}$ along the MA, as well as an increasing velocity dispersion profile.

PGC 044257: the data indicate that the velocity dispersion might increase with radius, although the large scatter and the small number of bins make it difficult to assess the slope.

PGC 071807: no significant rotation or substructure can be seen in this galaxy.

PGC 072804: the surface brightness profile was published by Malumuth \& Kirshner (1985). The radial velocity profile of this galaxy shows rotation of the order of $50 \mathrm{~km} \mathrm{~s}^{-1}$ along the MA.

UGC 02232: this galaxy has an extended envelope and a large number of objects in the vicinity. The HST imaging was published by Laine et al. (2003). No significant rotation or clear substructure is detected.

UGC 05515: the surface brightness profile was published by Schombert (1986). The radial velocity profile shows a KDC in the centre of this galaxy.

UGC 10143: this galaxy has a chain of companions. The surface brightness profile was published by Schombert (1986) and the $H S T$ imaging by Laine et al. (2003). No significant rotation or substructure can be inferred from the radial kinematic profiles of this galaxy.

\section{DISCUSSION}

(i) Five out of 41 BCGs (ESO 349-010, ESO 444-046, ESO 552-020, NGC 3311, PGC 026269) were found to have a positive velocity dispersion gradient.

The radial kinematic studies done so far on early-type galaxies are mostly limited to normal ellipticals, for which flat or decreasing velocity dispersion profiles are found. The majority of the results previously obtained for very small samples of BCGs are similar to those of normal ellipticals. Fisher et al. (1995a) found one galaxy (IC 1101) in their sample of 13 BCGs with a positive velocity dispersion gradient. Carter et al. (1999) found one (NGC 6166) of their sample of three BCGs to have a positive velocity dispersion gradient, although Fisher et al. (1995a) did not find it for this galaxy. Brough et al. (2007) found negative velocity dispersion gradients in five out of their sample of six brightest cluster and group galaxies (the other one had a zero velocity dispersion gradient).

Both IC 1101 and NGC 6166 form part of the sample of BCGs studied here, but in both cases the measured velocity dispersion profiles do not reach the radius achieved in the much smaller samples in the above-mentioned studies. Thus, the positive velocity dispersion gradient could not be confirmed for IC 1101 or NGC 6166 (see Figs A3 and A4). However, five other BCGs were found to have a positive velocity dispersion gradient, though admittedly the slope is marginally positive in some cases. If these positive velocity dispersion gradients are not caused by systematic errors in the various data reduction or velocity dispersion measurements by different authors, then they imply a rising mass-to-light ratio. (ii) At least 12 BCGs (NGC 3842, NGC 4889, NGC 7647, ESO 488-027, IC 5358, MCG-02-12-039, NGC 1713, NGC 2832, NGC 4839, NGC 6269, NGC 7649 and UGC 05515) show clear velocity substructure in their profiles.

From studies of elliptical galaxies in high-density environments (e.g. Koprolin \& Zeilinger 2000), the incidence of KDCs is observed to be about 33 per cent, rising to 50 per cent when projection effects are considered. Hau \& Forbes (2006) find KDCs in 40 per cent of their isolated elliptical galaxies. For BCGs, we have found at least 12 of the 41 BCGs show clear velocity substructure, amounting to 29 per cent of the sample (intermediate- and minor-axis data included).

KDCs can be the result of a merger event (Koprolin \& Zeilinger 2000), but can also occur when the galaxy is triaxial and supports different orbital types in the core and main body (Statler 1991). The fact that the incidence of KDCs in BCGs compares with that found for normal elliptical galaxies in high-density environments suggests that the two classes share the same fraction of galaxies with triaxial shapes.

(iii) NGC 6034 and 7768 possess significant rotation (134 and $114 \mathrm{~km} \mathrm{~s}^{-1}$, respectively) along the MA. Several other BCGs show rotation that is $>40 \mathrm{~km} \mathrm{~s}^{-1}$ and more than three times the standard error: ESO 346-003, GSC555700266 and NGC 4839 (MA spectra); ESO 488-027, IC 5358 and UGC 05515 (intermediate axis spectra), as do the two elliptical galaxies NGC 4946 (MA spectra) and NGC 6047 (intermediate axis spectra).

Carter et al. (1999) found small rotation along the MA at large radii (30-40 arcsec) for their sample of three BCGs, which is consistent with the nearly complete lack of rotation found near the centres of the sample of 13 BCGs by Fisher et al. (1995a). According to Fisher et al. (1995a), the lack of rotation found in samples of BCGs is in agreement with the expectation of declining importance of rotation with increasing luminosity for elliptical galaxies. The lack of rotation is also compatible with the idea that these objects formed through dissipationless mergers (Boylan-Kolchin et al. 2006). The remnants left by mergers with or without dissipation are expected to differ in their kinematical structure. In a merger which involves gas-rich galaxies, the gas will form a disc. After the gas has been removed from the system at the end of the merger (through ejection and converted into stars), the remnant will show rotation (Bournaud, Jog \& Combes 2005). Whereas in a merger where dissipationless processes dominate, the remnant will show little or no rotation (Naab \& Burkert 2003; Cox et al. 2006). In this study, clear rotation above $100 \mathrm{~km} \mathrm{~s}^{-1}$ was found for NGC 6034 and 7768, while most BCGs showed little or no rotation. This kinematical differentiation (the existence of slow and fast rotators) in early-type galaxies is also clearly visible in the SAURON data presented by Emsellem et al. (2007).

The amount of flattening that is expected due to the rotation in a galaxy depends on the balance between ordered and random motions, and this can be quantified using the anisotropy parameter. The rotation of elliptical galaxies is conventionally expressed as the anisotropy parameter, defined as $\left(V_{\max } / \sigma_{0}\right)^{*}=$ $\left(V_{\max } / \sigma_{0}\right) / \sqrt{\epsilon / 1-\epsilon}$ (Kormendy 1982), where the rotational velocity $V_{\max }$ is measured as in Section 5 and the central velocity dispersion $\sigma_{0}$ is taken as the measurement for the central velocity dispersion in Table 4. A value of $\left(V_{\max } / \sigma_{0}\right)^{*} \approx 1$ would be expected if a galaxy is flattened by rotation. The anisotropy parameter $\left(V_{\max } / \sigma_{0}\right)^{*}$ can be used to separate galaxies that are rotationally supported from those that are supported by $\sigma$ anisotropy where the 
Table 4. Central velocity dispersions $(\sigma)$ and radial velocities $(V)$ of the galaxies. The measurement and determination of $V_{\max }$ and $\left(V_{\max } / \sigma_{0}\right)^{*}$ are described in Section 6.

\begin{tabular}{|c|c|c|c|c|}
\hline Galaxy & $\begin{array}{c}\sigma \\
\left(\mathrm{km} \mathrm{s}^{-1}\right) \\
\text { Observed }\end{array}$ & $\begin{array}{c}V \\
\left(\mathrm{~km} \mathrm{~s}^{-1}\right) \\
\text { Observed }\end{array}$ & $\begin{array}{c}V_{\max } \\
\left(\mathrm{km} \mathrm{s}^{-1}\right)\end{array}$ & $\log \left(V_{\max } / \sigma_{0}\right)^{*}$ \\
\hline ESO 146-028 & $299 \pm 3$ & $12061 \pm 3$ & $14 \pm 18$ & $-1.2 \pm 0.9$ \\
\hline ESO 303-005 & $276 \pm 5$ & $14526 \pm 4$ & $23 \pm 17$ & $-0.8 \pm 0.4$ \\
\hline ESO 346-003 & $226 \pm 4$ & $8459 \pm 9$ & $51 \pm 13$ & $-0.3 \pm 0.1$ \\
\hline ESO 349-010 & $282 \pm 3$ & $14420 \pm 3$ & $46 \pm 19$ & $-0.8 \pm 0.4$ \\
\hline ESO 444-046 & $292 \pm 3$ & $13741 \pm 3$ & $26 \pm 16$ & $-0.9 \pm 0.4$ \\
\hline ESO 488-027 & $248 \pm 2$ & $11754 \pm 4$ & $53 \pm 12$ & $-0.3 \pm 0.1$ \\
\hline ESO 552-020 & $229 \pm 3$ & $9128 \pm 5$ & $20 \pm 19$ & $-1.0 \pm 0.8$ \\
\hline GSC555700266 & $312 \pm 9$ & $20059 \pm 6$ & $49 \pm 14$ & $-0.6 \pm 0.2$ \\
\hline IC 1101 & $378 \pm 5$ & $22585 \pm 9$ & $41 \pm 31$ & $-1.0 \pm 0.8$ \\
\hline IC 1633 & $400 \pm 2$ & $7061 \pm 2$ & $20 \pm 17$ & $-1.0 \pm 0.4$ \\
\hline IC 4765 & $286 \pm 5$ & $4403 \pm 6$ & $50 \pm 28$ & $-0.8 \pm 0.6$ \\
\hline IC 5358 & $243 \pm 3$ & $8539 \pm 9$ & $55 \pm 13$ & $-0.7 \pm 0.3$ \\
\hline Leda094683 & $332 \pm 5$ & $22748 \pm 5$ & $32 \pm 26$ & $-0.9 \pm 0.6$ \\
\hline MCG-02-12-039 & $271 \pm 5$ & $9648 \pm 3$ & $59 \pm 28$ & $-0.3 \pm 0.3$ \\
\hline NGC 1399 & $371 \pm 3$ & $1406 \pm 2$ & $24 \pm 11$ & $-0.6 \pm 0.1$ \\
\hline NGC 1713 & $251 \pm 2$ & $4472 \pm 2$ & $30 \pm 11$ & $-0.5 \pm 0.1$ \\
\hline NGC 2832 & $364 \pm 4$ & $6841 \pm 4$ & $64 \pm 22$ & $-0.4 \pm 0.2$ \\
\hline NGC 3311 & $196 \pm 2$ & $3709 \pm 2$ & $25 \pm 9$ & $-0.6 \pm 0.2$ \\
\hline NGC 3842 & $287 \pm 5$ & $6211 \pm 2$ & $11 \pm 13$ & $-1.2 \pm 0.8$ \\
\hline NGC 4839 & $278 \pm 2$ & $7380 \pm 2$ & $44 \pm 14$ & $-0.8 \pm 0.3$ \\
\hline NGC 4874 & $267 \pm 4$ & $6953 \pm 2$ & $15 \pm 1$ & $-0.4 \pm 0.1$ \\
\hline NGC 4889 & $380 \pm 4$ & $6266 \pm 5$ & $40 \pm 19$ & $-0.8 \pm 0.3$ \\
\hline NGC 4946 & $201 \pm 2$ & $3148 \pm 9$ & $62 \pm 9$ & $-0.2 \pm 0.1$ \\
\hline NGC 6034 & $325 \pm 4$ & $10113 \pm 28$ & $134 \pm 15$ & $-0.2 \pm 0.1$ \\
\hline NGC 6047 & $230 \pm 2$ & $9031 \pm 8$ & $59 \pm 16$ & $-0.4 \pm 0.2$ \\
\hline NGC 6086 & $318 \pm 5$ & $9483 \pm 2$ & $13 \pm 18$ & $-1.2 \pm 0.9$ \\
\hline NGC 6160 & $266 \pm 3$ & $9428 \pm 3$ & $12 \pm 18$ & $-1.3 \pm 1.4$ \\
\hline NGC 6166 & $310 \pm 10$ & $9100 \pm 2$ & $31 \pm 20$ & $-0.7 \pm 0.4$ \\
\hline NGC 6173 & $304 \pm 3$ & $8766 \pm 2$ & $24 \pm 16$ & $-0.9 \pm 0.4$ \\
\hline NGC 6269 & $343 \pm 5$ & $10363 \pm 3$ & $26 \pm 35$ & $-0.8 \pm 0.7$ \\
\hline NGC 7012 & $240 \pm 3$ & $8653 \pm 3$ & $24 \pm 16$ & $-0.9 \pm 0.6$ \\
\hline NGC 7597 & $264 \pm 8$ & $10907 \pm 5$ & $63 \pm 20$ & $0.2 \pm 0.1$ \\
\hline NGC 7647 & $271 \pm 5$ & $11842 \pm 7$ & $36 \pm 14$ & $-0.9 \pm 0.4$ \\
\hline NGC 7649 & $250 \pm 5$ & $12258 \pm 5$ & $41 \pm 14$ & $-0.6 \pm 0.2$ \\
\hline NGC 7720 & $409 \pm 5$ & $9034 \pm 5$ & $50 \pm 18$ & $-0.6 \pm 0.2$ \\
\hline NGC 7768 & $272 \pm 5$ & $7875 \pm 24$ & $114 \pm 11$ & $-0.1 \pm 0.1$ \\
\hline PGC 026269 & $222 \pm 11$ & $15816 \pm 8$ & $51 \pm 20$ & $0.2 \pm 0.1$ \\
\hline PGC 044257 & $247 \pm 9$ & $14014 \pm 6$ & $20 \pm 16$ & $-1.2 \pm 1.0$ \\
\hline PGC 071807 & $315 \pm 3$ & $17911 \pm 5$ & $16 \pm 13$ & $-1.5 \pm 1.2$ \\
\hline PGC 072804 & $311 \pm 5$ & $22433 \pm 4$ & $50 \pm 24$ & $-0.4 \pm 0.2$ \\
\hline UGC 02232 & $314 \pm 4$ & $14208 \pm 3$ & $42 \pm 18$ & $0.0 \pm 0.1$ \\
\hline UGC 05515 & $362 \pm 4$ & $13103 \pm 7$ & $56 \pm 18$ & $-0.4 \pm 0.1$ \\
\hline UGC 10143 & $262 \pm 2$ & $10344 \pm 5$ & $19 \pm 11$ & $-1.1 \pm 0.5$ \\
\hline
\end{tabular}

value is substantially less than unity. The division occurs at about $\left(V_{\max } / \sigma_{0}\right)^{*}=0.7$ (Bender, Burstein \& Faber 1992).

Fig. 8 shows the anisotropy parameter as a function of galaxy $B$-band luminosity, where the above-mentioned division is indicated by the horizontal dashed line. Note that the errors indicated are only propagated from the errors on the velocity measurements taken to be the extreme radial velocity points, and the errors on the central velocity dispersion. They do not take into account the general uncertainties involved in determining the most extreme velocity measurements. Therefore, care has to be taken when interpreting the individual points on the diagram. The only notable BCG data point that lies significantly above the dashed line is for the galaxy PGC 026269, which possesses moderate rotation $\left(51 \mathrm{~km} \mathrm{~s}^{-1}\right)$ and

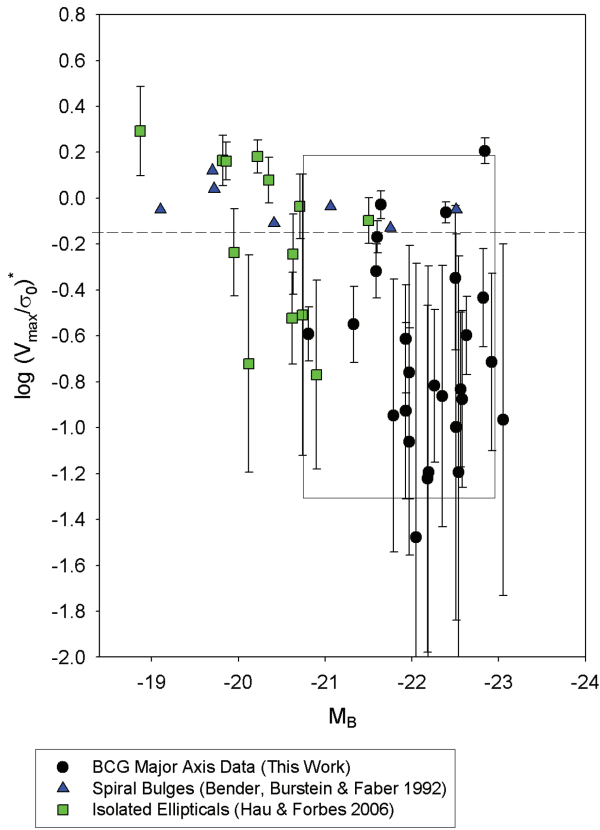

Figure 8. The anisotropy-luminosity diagram. The horizontal dashed line separates the rotationally supported galaxies from the anisotropic galaxies as described in the text. Only the BCGs for which MA spectra were taken (within $10^{\circ}$ ) are plotted. The box plotted in the figure outlines the region containing data on giant ellipticals from Bender et al. (1992).

surprisingly low central velocity dispersion $\left(222 \mathrm{~km} \mathrm{~s}^{-1}\right)$. However, the ellipticity of this galaxy is zero and it is not rotationally supported.

Another factor that complicates the dynamical interpretation of individual points is that the observed ellipticity is a global property of the galaxy, whereas the kinematic measurements taken here only reflect the kinematics along the axis where the slit was placed, and only close to the centre of the galaxy. For example, a disc component may dominate the measured kinematics but will have little effect on the ellipticity, making the galaxy appear to rotate faster than its global ellipticity would suggest (Merrifield 2004).

For comparison, the sample of isolated ellipticals from Hau \& Forbes (2006) is also plotted in Fig. 8. Of this sample, 11 galaxies were observed along the MA, and the central velocity dispersions were derived from the bins closest to the galaxy cores. The BCGs show less rotational support than the isolated elliptical galaxies as a class. Spiral bulge (typically rotationally supported) and giant elliptical data from Bender et al. (1992) are also plotted. Their central velocity dispersions were derived over the whole half-light radii of the galaxies. Even though large rotation velocities were found for a few individual cases, the BCGs are consistent with the general trend for very massive galaxies.

\section{SUMMARY}

In this paper, we have presented the largest optical spectroscopic sample of BCGs with radial information to date. We have derived the rotational velocity and velocity dispersion profiles for 41 BCGs. We find clear rotation curves for a number of galaxies for which we have obtained MA spectra and two galaxies with rotational velocities exceeding $100 \mathrm{~km} \mathrm{~s}^{-1}$. However, because of the generally large central velocity dispersions, the BCG data are consistent with 
the trend for very massive elliptical galaxies on the anisotropyluminosity diagram. At least 29 per cent of the galaxies show very clear velocity substructure.

A future paper will be devoted to the stellar population properties of this galaxy sample. Despite the undeniably special nature of BCGs due to their extreme morphological properties and locations, the kinematic properties investigated here (rotation and incidence of velocity substructure) seem normal compared with their ordinary giant elliptical counterparts.

\section{ACKNOWLEDGMENTS}

SIL thanks the South African National Research Foundation and the University of Central Lancashire for a Stobie-SALT Scholarship. We gratefully acknowledge Bryan Miller (PI of the Gemini programmes to collect the Lick star spectra) and J.J Gonzalez for useful discussions. We also thank the anonymous referee for constructive comments, which contributed to the improvement of this paper. PSB is supported by a Marie Curie Intra-European Fellowship within the 6th European Community Framework Programme.

Based on the observations obtained on the WHT and Gemini North and South telescopes (Gemini programme numbers GS2006B-Q-71, GN-2006B-Q-88, GS-2007A-Q-73, GN-2007A-Q123, GS-2007B-Q-43 and GN-2007B-Q-101). The WHT is operated on the island of La Palma by the Royal Greenwich Observatory at the Observatorio del Roque de los Muchachos of the Instituto de Astrofísica de Canarias. The Gemini Observatory is operated by the Association of Universities for Research in Astronomy, Inc., under cooperative agreement with the NSF on behalf of the Gemini Partnership: the National Science Foundation (USA), the Science and Technology Facilities Council (UK), the National Research Council (Canada), CONICYT (Chile), the Australian Research Council (Australia), CNPq (Brazil) and CONICET (Argentina). This research has made use of the NED which is operated by the Jet Propulsion Laboratory, California Institute of Technology and the HyperLeda catalogue HyperLEDA which is an extended version of the Lyon-Meudon Extragalactic Data base operated by the Centre de Recherche Astronomique de Lyon.

\section{REFERENCES}

Adami C., Biviano A., Durret F., Mazure A., 2005, A\&A, 443, 17

Andreon S., Davoust E., Michard R., Nieto J. L., Poulain P., 1996, A\&AS, 116,429

Aragon-Salamanca A., Baugh C. M., Kauffmann G., 1998, MNRAS, 297, 427

Baier F. W., Wipper H., 1995, AN, 316, 319

Bautz L. P., Morgan W. W., 1970, BAAS, 2, 294 (BM)

Bender R., Burstein D., Faber S. M., 1992, ApJ, 399, 462

Bournaud F., Jog C. J., Combes F., 2005, A\&A, 437, 69

Boylan-Kolchin M., Ma C., Quataert E., 2006, MNRAS, 369, 1081

Brough S., Collins C. A., Burke D. J., Mann R. G., Lynam P. D., 2002, MNRAS, 329, 533

Brough S., Proctor R., Forbes D. A., Couch W. J., Collins C. A., Burke D. J., Mann R. G., 2007, MNRAS, 378, 1507

Burstein D., Faber S. M., Gaskell C. M., Krumm N., 1984, ApJ, 287, 586

Cardiel N., 1999, PhD thesis, Universidad Complutense de Madrid

Cardiel N., Gorgas J., Aragon-Salamanca A., 1998, MNRAS, 298, 977

Carter D., Metcalfe N., 1980, MNRAS, 191, 325

Carter D., Bridges T. J., Hau G. K. T., 1999, MNRAS, 307, 131

Collins C. A., Mann R. G., 1998, MNRAS, 297, 128

Cowie L. L., Binney J., 1977, ApJ, 215, 723

Cox T. J., Dutta S. N., Di Matteo T., Hernquist L., Hopkins P. F., Robertson B., Springel V., 2006, ApJ, 650, 791
De Vaucouleurs G., 1948, JO, 31, 113

De Lucia G., Blaizot J., 2007, MNRAS, 375, 2

De Lucia G., Springer V., White S. D. M., Croton D., Kauffmann G., 2006, MNRAS, 366, 499

Dressler A., 1984, ApJ, 281, 512

Edwards L. O. V., Hudson M. J., Balogh M. L., Smith R. J., 2007, MNRAS, 379,100

Emsellem E. et al., 2007, MNRAS, 379, 401

Faber S. M., Friel E. D., Burstein D., Gaskell C. M., 1985, ApJS, 57, 711

Fisher D., Illingworth G., Franx M., 1995a, ApJ, 438, 539

Fisher D., Franx M., Illingworth G., 1995b, ApJ, 448, 119

Gallagher J. S., Ostriker J. P., 1972, AJ, 77, 288

Giacintucci S., Venturi T., Murgia M., Dallacasa D., Athreya R., Bardelli S., Mazzotta P., Saikia D. J., 2007, A\&A, 476, 99

González J. J., 1993, PhD thesis, Univ. California

Gorgas J., Efstathiou G., Aragon-Salamanca A., 1990, MNRAS, 245, 217

Hau G. K. T., Forbes D. A., 2006, MNRAS, 371, 633

Hill J. M., Oegerle W. R., 1992, A\&AS, 18, 1413

Hoessel J. G., Gunn J. E., Thuan T. X., 1980, ApJ, 241, 486

Jarrett T. H., Chester T., Cutri R., Schneider S. E., Huchra J. P., 2003, AJ, 125,525

Jordán A., Côté P., West M. J., Marzke R. O., Minniti D., Rejkuba M., 2004, AJ, 127, 24

Kormendy J., 1982, in Martinet L., Major M., eds, Morphology and Dynamics of Galaxies. Geneva Observatory, Sauverny, p. 115

Koprolin W., Zeilinger W., 2000, A\&A, 145, 71

Laine S., van der Marel R. P., Lauer T. R., Postman M., O’Dea C. P., Owen F. N., 2003, AJ, 125, 478

Lauer T. R., 1988, ApJ, 325, 49

Liu F. S., Xia X. Y., Mao S., Wu H., Deng Z. G., 2008, MNRAS, 385, 23

Longo G., Zaggio S. R., Brusarello G., Richter G., 1994, A\&AS, 105, 433

McNamara B. R., O’Connell R. W., 1992, ApJ, 393, 579

McNamara B. R., Wise M., Sarazin C. L., Jannuzi B. T., Elston R., 1996, ApJ, 466, L9

Malumuth E. M., Kirshner R. P., 1985, ApJ, 291, 8

Matthews T. A., Morgan W. W., Schmidt M., 1964, ApJ, 140, 35

Mehlert D., Saglia R. P., Bender R., Wegner G., 2000, A\&AS, 141, 449

Merrifield M., 2004, MNRAS, 353, L13

Merritt D., 1983, ApJ, 264, 24

Naab T., Burkert A., 2003, ApJ, 597, 893

Neumann D. M. et al., 2001, A\&A, 365, 74

Oegerle W. R., Hill J. M., 2001, AJ, 122, 2858

Oemler A., 1976, ApJ, 209, 693

Ostriker J. P., Hausman M. A., 1977, ApJ, 217, 125

Ostriker J. P., Tremaine S. D., 1975, ApJ, 202, 113

Patel P., Maddox S., Pearce F. R., Aragón-Salamanca A., Conway E., 2006 MNRAS, 370, 851

Peletier R. F., Davies R. L., Illingworth G. D., Davis L. E., Cawson M., 1990, AJ, 100, 1091

Postman M., Lauer T. R., 1995, ApJ, 440, 28

Proctor R. N., Forbes D. A., Forestell A., Gebhardt K., 2005, MNRAS, 362 , 857

Prugniel Ph., Simien F., 1996, A\&A, 309, 749

Rines K., Geller M. J., Diaferio A., Mahdavi A., Mohr J. J., Wegner G., 2002, AJ, 124, 1266

Romeo A. D., Napolitano N. R., Covone G., Sommer-Larsen J., AntonuccioDelogu V., Capacciolo M., 2008, MNRAS, 389, 13

Rood H. J., Sastry G. N., 1971, PASP, 83, 313 (RS)

Salomé P., Combes F., 2003, A\&A, 412, 657

Sánchez-Blázquez P., Gorgas J., Cardiel N., González J. J., 2006, A\&A, 457,787

Sarazin C. L., 1988, X-ray Emission from Clusters of Galaxies. Cambridge Univ. Press, Cambridge

Sargent W., Schechter P., Boksenberg A., Shortridge K., 1977, ApJ, 212, 326

Schombert J. M., 1986, ApJS, 60, 603

Schombert J. M., 1987, ApJS, 64, 643

Schombert J. M., 1988, ApJ, 328, 475 
Seigar M. S., Graham A. W., Jerjen H., 2007, MNRAS, 378, 1575

Statler T. S., 1991, AJ, 102, 882

Struble M. F., Rood H. J., 1987, ApJS, 63, 555

Tonry J. L., 1984, ApJ, 279, 13

Tonry J. L., 1985, AJ, 90, 2431

Torlina L., De Propris R., West M. J., 2007, ApJ, 660, L97

Van der Marel R. P., Franx M., 1993, ApJ, 407, 525

Vitores A. G., Zamorano J., Rego M., Alonso O., Gallego J., 1996, A\&AS, 118,7

Von der Linden A., Best P. N., Kauffmann G., White S. D. M., 2007, MNRAS, 379, 867

West M. J., 1989, ApJ, 344, 535

Whiley I. M. et al., 2008, MNRAS, 387, 1253

Worthey G., Ottaviani D. L., 1997, ApJS, 111, 377

Worthey G., Faber S. M., González J. J., Burstein D., 1994, ApJS, 94, 687

Yamada T., Koyama Y., Nakata F., Kajisawa M., Tanaka I., Kodama T., Okamura S., De Propis R., 2002, ApJ, 577, 89

\section{APPENDIX A: KINEMATIC PROFILES: COMPARISON WITH LITERATURE}

The kinematic radial profiles of some of the galaxies could be compared to previous measurements in the literature. Four comparisons are shown in Figs A1 to A4. Two of the Coma cluster cD galaxies (NGC 4839 and NGC 4889) were compared to the data from Fisher et al. (1995a) and Mehlert et al. (2000). The third Coma cD (NGC 4874) was not compared as it was observed at a different slit PA than in the other studies. No systematic differences are found (within the error bars) for the measurements of this study compared to the previous studies. The velocity dispersion measurements of NGC 4889 in Fisher et al. (1995a) are lower than the other studies. This discrepancy was already reported in Mehlert et al. (2000). NGC 6166 was compared to Fisher et al. (1995a) and agree within the errors. IC

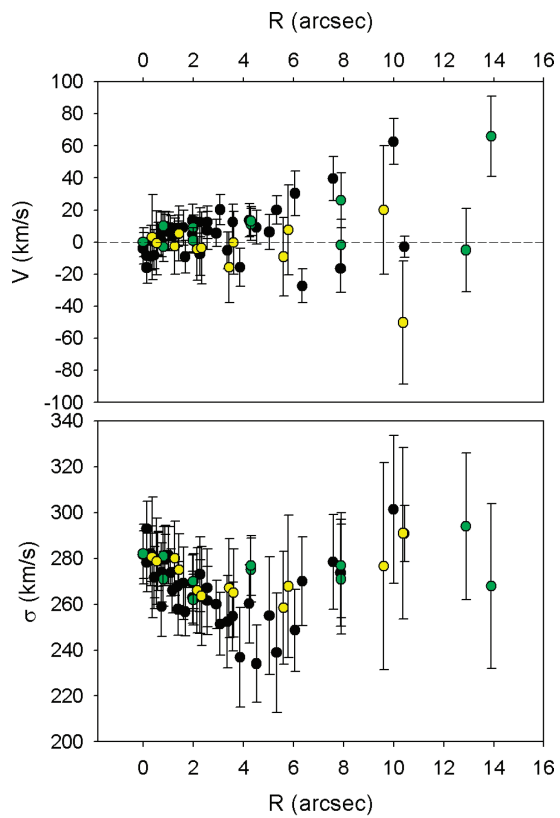

$$
\begin{array}{ll}
\text { - } & \text { This study } \\
\text { - } & \text { Fisher et al. (1995a) } \\
\text { - } & \text { Mehlert et al. (2000) }
\end{array}
$$

Figure A1. NGC 4839. Kinematic profile compared to previous literature. The data are folded with respect to the centre of the galaxy, and the radial velocities are given as relative to the central radial velocity.

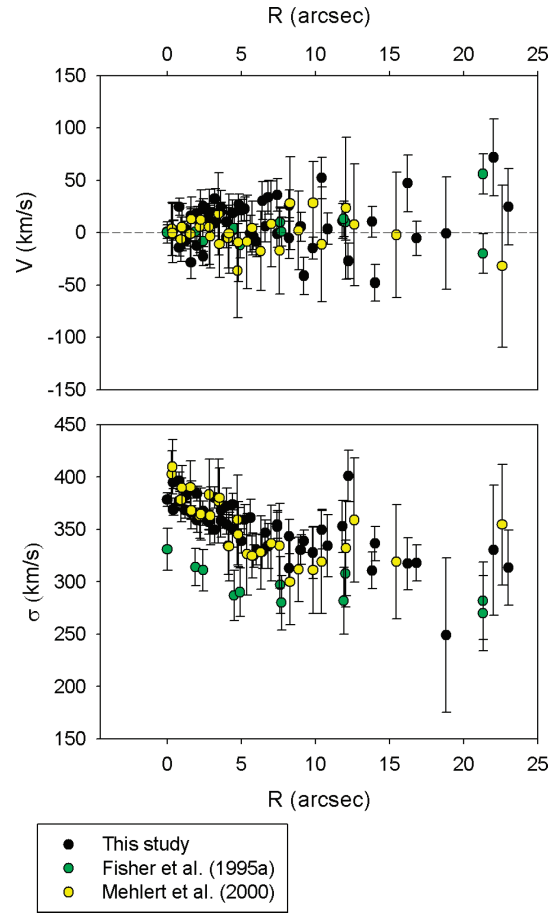

Figure A2. NGC 4889. Kinematic profile compared to the previous literature.

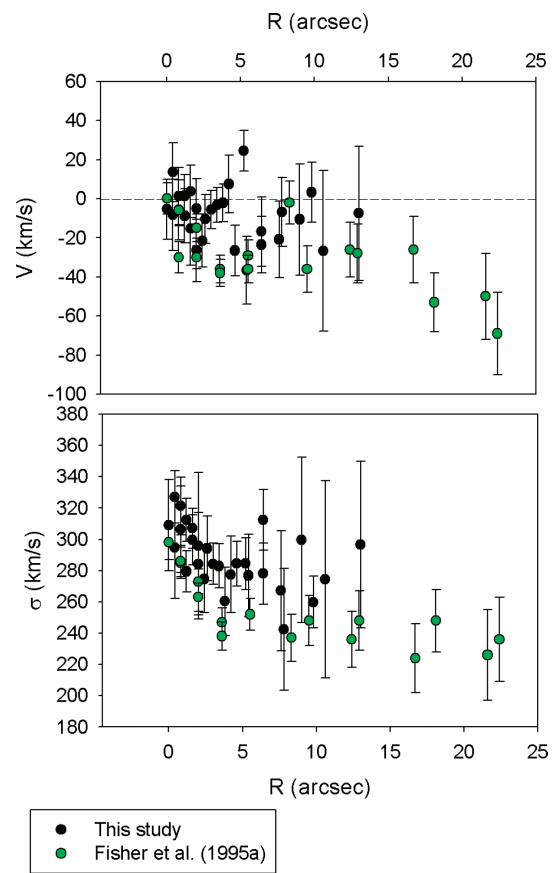

Figure A3. NGC 6166. Kinematic profile compared to the previous literature.

1101 was compared to Fisher et al. (1995a), who found the velocity dispersion profile to be increasing. As can be seen from Fig. A4, the velocity dispersion profile measured here does not extend out to the same radius as that from Fisher et al. (1995a), but the central measurements are in good agreement. 

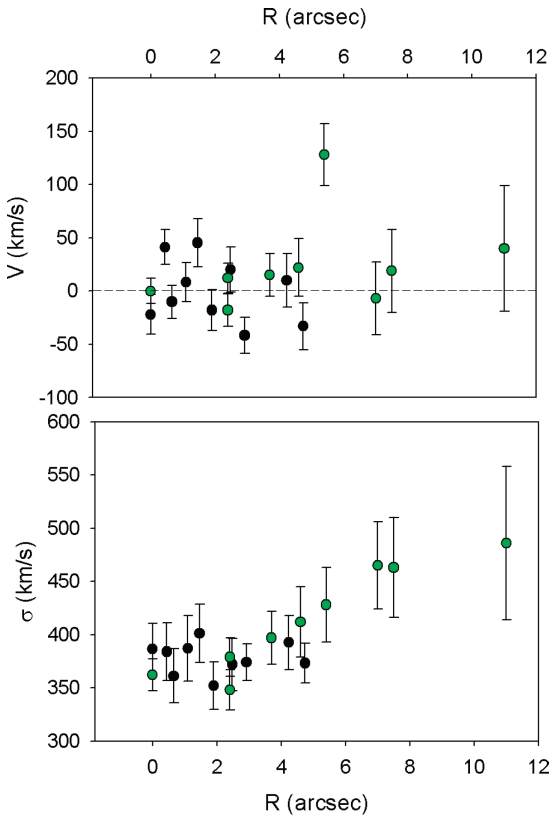

- This study

- Fisher et al (1995a)

Figure A4. IC 1101. Kinematic profile compared to previous literature.

\section{APPENDIX B：SUPPORTING INFORMATION}

Additional supporting information may be found in the online version of this article.

Table B1. Radial kinematics of ESO 146-028.

Table B2. Radial kinematics of ESO 303-005.

Table B3. Radial kinematics of ESO 346-003.

Table B4. Radial kinematics of ESO 349-010.

Table B5. Radial kinematics of ESO 444-046.

Table B6. Radial kinematics of ESO 488-027.

Table B7. Radial kinematics of ESO 552-020.

Table B8. Radial kinematics of GSC555700266.

Table B9. Radial kinematics of IC 1101.

Table B10. Radial kinematics of IC 1633.
Table B11. Radial kinematics of IC 4765.

Table B12. Radial kinematics of IC 5358.

Table B13. Radial kinematics of Leda094683.

Table B14. Radial kinematics of MCG-02-12-039.

Table B15. Radial kinematics of NGC 1399.

Table B16. Radial kinematics of NGC 1713.

Table B17. Radial kinematics of NGC 2832.

Table B18. Radial kinematics of NGC 3311.

Table B19. Radial kinematics of NGC 3842.

Table B20. Radial kinematics of NGC 4839.

Table B21. Radial kinematics of NGC 4874.

Table B22. Radial kinematics of NGC 4889.

Table B23. Radial kinematics of NGC 4946.

Table B24. Radial kinematics of NGC 6034.

Table B25. Radial kinematics of NGC 6047.

Table B26. Radial kinematics of NGC 6086.

Table B27. Radial kinematics of NGC 6160.

Table B28. Radial kinematics of NGC 6166.

Table B29. Radial kinematics of NGC 6173.

Table B30. Radial kinematics of NGC 6269.

Table B31. Radial kinematics of NGC 7012.

Table B32. Radial kinematics of NGC 7597.

Table B33. Radial kinematics of NGC 7647.

Table B34. Radial kinematics of NGC 7649.

Table B35. Radial kinematics of NGC 7720.

Table B36. Radial kinematics of NGC 7768.

Table B37. Radial kinematics of PGC 026269.

Table B38. Radial kinematics of PGC 044257.

Table B39. Radial kinematics of PGC 071807.

Table B40. Radial kinematics of PGC 072804.

Table B41. Radial kinematics of UGC 02232.

Table B42. Radial kinematics of UGC 05515.

Table B43. Radial kinematics of UGC 10143.

Please note: Wiley-Blackwell are not responsible for the content or functionality of any supporting information supplied by the authors. Any queries (other than missing material) should be directed to the corresponding author for the article.

This paper has been typeset from a $\mathrm{T}_{\mathrm{E}} \mathrm{X} / \mathrm{LT}_{\mathrm{E}} \mathrm{X}$ file prepared by the author. 